A\&A 654, A79 (2021)

https://doi.org/10.1051/0004-6361/202140790

\title{
Quasar clustering at redshift 6
}

\author{
J. Greiner ${ }^{1}$, J. Bolmer ${ }^{1}$, R. M. Yates ${ }^{2,3}$, M. Habouzit ${ }^{4,5}$, E. Bañados ${ }^{4}$, P. M. J. Afonso ${ }^{6}$, and P. Schady ${ }^{7}$ \\ 1 Max-Planck Institut für extraterrestrische Physik, Giessenbachstr. 1, 85748 Garching, Germany \\ e-mail: jcg@mpe.mpg.de \\ 2 Max-Planck Institut für Astrophysik, Karl-Schwarzschildstr. 1, 85748 Garching, Germany \\ 3 University of Surrey, Guildford, Surrey GU2 7XH, UK \\ 4 Max-Planck Institut für Astronomie, Königstuhl 17, 69117 Heidelberg, Germany \\ 5 Zentrum für Astronomie der Universität Heidelberg, ITA, Albert-Ueberle-Str. 2, 69120 Heidelberg, Germany \\ 6 American River College, Physics \& Astronomy Dpt., 4700 College Oak Drive, Sacramento, CA 95841, USA \\ 7 Department of Physics, University of Bath, Bath BA2 7AY, UK
}

Received 12 March 2021 / Accepted 20 July 2021

\begin{abstract}
Context. Large-scale surveys over the last years have revealed about 300 quasi-stellar objects (QSOs) at redshifts above 6. Follow-up observations have identified surprising properties, such as the very high black hole $(\mathrm{BH})$ masses, spatial correlations with surrounding cold gas of the host galaxy, and high CIV -MgII velocity shifts. In particular, the discovery of luminous high-redshift quasars suggests that at least some BHs likely have high masses at birth and grow efficiently.

Aims. Our aim is to quantify quasar pairs at high redshift for a large sample of objects. This provides a new key constraint on a combination of parameters related to the origin and assembly for the most massive BHs: formation efficiency and clustering, growth efficiency, and the relative contribution of BH mergers.

Methods. We observed 116 spectroscopically confirmed QSOs around redshift 6 with the simultaneous seven-channel imager Gammaray Burst Optical/Near-infrared Detector in order to search for companions. Applying colour-colour cuts identical to those which led to the spectroscopically confirmed QSOs, we performed Le PHARE fits to the 26 best QSO pair candidates, and obtained spectroscopic observations for 11 of them.

Results. We do not find any QSO pair with a companion brighter than $M_{1450}(\mathrm{AB})<-26$ mag within our $0.1-3.3 h^{-1} \mathrm{cMpc}$ search radius, in contrast to the serendipitous findings in the redshift range 4-5. However, a small fraction of such pairs at this luminosity and redshift is consistent with indications from present-day cosmological-scale galaxy evolution models. In turn, the incidence of L- and T-type brown dwarfs, which occupy a similar colour space to $z \sim 6$ QSOs, is higher than expected, by a factor of 5 and 20 , respectively.
\end{abstract}

Key words. early Universe - galaxies: active - quasars: general - stars: low-mass - brown dwarfs

\section{Introduction}

The clustering of quasars provides one of the most important observational constraints to probe their physical properties, their formation and evolution (Haiman \& Hui 2001), and the mass of their dark matter (DM) halos (Sheth et al. 2001). As easily detectable objects throughout the Universe, quasars trace the underlying dark matter distribution (e.g., Haehnelt \& Nusser August 1998), and thus provide a powerful test of hierarchical structure formation theory (e.g., Fang 1989). How this structure formation in the early Universe proceeds in detail is far from understood. How many massive galaxies form in a single massive dark matter halo? Do all of these massive galaxies have a massive black hole (BH)? Do all these BHs accrete from the rich gas reservoir, and what are the duty cycles of QSO activity? Do massive galaxies form sub-structures and/or multiple massive BHs?

Particularly pressing issues over the last years are the formation channels and efficiencies of massive $\mathrm{BHs}$, and their growth rates with cosmic time (e.g., Johnson et al. 2013; Valiante et al. 2017, 2018; Inayoshi et al. 2020; Kroupa et al. 2020). Quantifying the number of QSO pairs at high redshift is one observational avenue to constrain a combination of parameters of
$\mathrm{BH}$ formation and $\mathrm{BH}$ growth rate. High- $z$ QSOs can be as massive as the most massive BHs found in the local Universe (e.g., Bañados et al. 2018; Yang et al. 2020; Wang et al. 2021), and so must have grown several orders of magnitude in less than 800 Myr. Black hole formation mechanisms predict the formation of BH seeds with masses of a few $\sim 10^{5-6} M_{\odot}$ at most (Volonteri 2010; Inayoshi et al. 2020). If the growth of quasars was mostly through gas accretion, then they must have grown at the Eddington rate for a large fraction of their lives. Finding QSO pairs, or the absence of them, can help us understand if $\mathrm{BH}$ mergers play a role in their growth, and if dense environments can sustain efficient growth for several QSOs in the same region of the sky or if baryonic processes (e.g., AGN feedback) prevent them from doing so (Wyithe et al. 2005; Kashikawa et al. 2007; Utsumi et al. 2010; Costa et al. 2014; Habouzit et al. 2019, for QSO feedback preventing galaxy formation and/or growth in their surroundings). Radiation from a QSO can impact a region from sub-Mpc to tens of Mpc, depending on the QSO mass, accretion rate, and properties of its surrounding environment, and could therefore also inhibit the accretion activity of nearby BHs. In addition, quantifying the number of QSO pairs also provides us with constraints on $\mathrm{BH}$ formation efficiency (i.e. whether $\mathrm{BH}$ formation takes place in a large number of halos), 
but also on whether the formation of massive seeds could be clustered. The existence of many pairs of QSOs could indeed imply that massive seeds can form close to each other. Constraints from QSO pairs on QSO origins and growth with time are likely degenerated, but are key to improving our understanding of these extreme objects.

Quasar clustering has been investigated at different scales and depths, among others using the Sloan Digital Sky Survey (SDSS) (Shen et al. 2007; Hennawi et al. 2010), the 2dF QSO Redshift and Dark Energy Survey (DES) (Porciani et al. 2007; Chehade et al. 2016), or the Canada-France-Hawaii Telescope Legacy Survey (CFHTLS) (McGreer et al. 2016). Studies at different depths for a given redshift range are important since a large luminosity dependence is theoretically predicted at higher redshifts (Hopkins et al. 2007). Clustering at large scales, sampling megaparsec or larger distances, is stronger at high redshifts $(z>3.5)$ than at low redshifts $(2.9<z<3.5)$ (Shen et al. 2007). Clustering at small scales, sampling the $10 \mathrm{kpc}-1 \mathrm{Mpc}$ environment, also increases from lower $(z \sim 3)$ to higher $(z \sim 4)$ redshift, but shows a much stronger amplitude on kiloparsec scales than on megaparsec scales (e.g., a factor 4 stronger at $z \sim 2$ ) (Eftekharzadeh et al. 2017).

Clustering measurements at high redshift $(z>3)$ come up against a problem: close quasar pairs are extremely rare. For comparison, the mean quasar separation at $z>3$ is $\sim 150 \mathrm{~h}^{-1} \mathrm{Mpc}$ (given the standard luminosity function of $\Phi \sim$ $10^{-6} \mathrm{Mpc}^{-1} \mathrm{mag}^{-1}$ for $M(\mathrm{AB})<-24$; e.g., Willott et al. 2010; Ross et al. 2013; Matsuoka et al. 2018; Kulkarni et al. 2019), and on small scales $(\lesssim 1 \mathrm{Mpc})$ the correlation function does not increase as fast as the volume decreases (Hennawi et al. 2010). However, direct observations of pairs might suggest differently; the three QSO pairs at the highest redshifts were all found serendipitously: (i) Schneider et al. (2000) reported a pair at $z=4.26$ with a separation of $33^{\prime \prime}$ (corresponding to $160 h^{-1} \mathrm{kpc}$ proper on the sky) which was found in the slit while spectroscopically confirming another QSO candidate; (ii) Djorgovski et al. (2003) discovered a $z=4.96$ quasar at a separation of 196" (or proper transverse separation of $0.9 h^{-1} \mathrm{Mpc}$ ) from a $z=5.02$ quasar discovered by Fan et al. (1999); and (iii) McGreer et al. (2016) reported a pair at $z=5.02$ with a separation of $21^{\prime \prime}\left(90 h^{-1} \mathrm{kpc}\right.$ ), discovered in a faint $z \sim 5$ quasar (but not pair) search down to $i=23 \mathrm{mag}$, and selected simultaneously with identical colour criteria. Given the relatively small sample sizes which these discoveries are based upon, they suggest that quasars are even stronger clustered at redshifts $>4$ on small scales. This in turn has been used to argue that feedback is very inefficient at high redshift (Willott et al. 2010; McGreer et al. 2016), since inefficient feedback would correspond to the correlation length to flatten out at high redshift, while concordant QSO and host halo mass growth would imply a sharply rising correlation length. In addition, merger models of galaxy formation predict a large fraction of binary QSOs, at least over some fraction of their life time (e.g., Volonteri et al. 2003a), with the fraction of binary (off-centre and dual) QSOs increasing with redshift (Volonteri et al. 2016). This state of knowledge, and the importance of the conclusions that can be drawn, certainly warrants further searches for clustering properties at high redshift.

Here we search within a sample of 116 spectroscopically confirmed QSOs at redshift 6 (collected from the compilation of Bañados et al. 2016) for a companion. Throughout this paper we use the best-fit Planck cosmological model with $\Omega_{m}=$ 0.286, $\Omega_{\Lambda}=0.714$, and $h=0.69$ (Planck Collaboration XIII 2016). At a mean sample redshift of 6 , an angular separation of $1^{\prime \prime}$ corresponds to a proper (comoving) transverse separation of $4 h^{-1} \mathrm{kpc}\left(28 h^{-1} \mathrm{kpc}\right)$. All magnitudes are in the $\mathrm{AB}$ system.

\section{Selection criteria, observations, and data analysis}

\subsection{Selection}

From the sample of 173 spectroscopically confirmed QSOs at $z>5.6$ compiled in Bañados et al. (2016) we selected all 144 sources south of Dec. $<+30^{\circ}$, thus being visible from La Silla (Chile). We added six objects from Mazzucchelli et al. (2017b), and two object from Jiang et al. (2016). For the observing campaigns we prioritised brighter sources, so that it was easier (less time consuming) to reach limiting magnitudes fainter than the spectroscopically confirmed QSOs. After weather and technical losses, we obtained useful new Gamma-ray Burst Optical/Nearinfrared Detector (GROND) observations for 77 sources, in addition to the 42 objects from the Bañados et al. (2016) sample, which had already been observed with GROND in 2013-2015 during the selection process towards their spectroscopic sample. Excluding in the following two QSOs at $z>6.6$, our sample of 116 QSOs spans redshifts in the range 5.6-6.6 and $z^{\prime}$-band brightness values in the range 18.6-23.1 mag.

\subsection{Imaging observations and data analysis}

\subsubsection{Strategy, data analysis and source detection}

Simultaneous imaging in $g^{\prime} r^{\prime} i^{\prime} z^{\prime} J H K_{\mathrm{s}}$ with GROND (Greiner et al. 2008) of the 77 new fields was performed at six different time slots: 2016 August 14 - September 3, 2016 October 11-25, 2017 January 24 - February 7, 2017 April 22 - May 2, 2017 September 18-21, and 2018 March 23. Since most fields have SDSS or Pan-STARRS coverage, no further observations for photometric calibration were obtained. The three remaining fields were calibrated against the SkyMapper Survey (DR1.1).

Since the field of view (FOV) of the four visual channels $g^{\prime} r^{\prime} i^{\prime} z^{\prime}$ is smaller than that of the near-infrared (NIR) channels (Fig. 1), the former define the search distance from the spectroscopically identified QSO to 80" distance. Thus, our maximum search radius for a QSO companion is $0.471 h^{-1} \mathrm{Mpc}$ proper or $3.3 h^{-1} \mathrm{Mpc}$ comoving (see Fig. 1). This accounts for the fact that most of the primary QSOs are not exactly centred in the GROND FOV.

The question to answer before the start of our observing campaign was how much deeper to look for a companion, relative to the prime QSO. We chose a 2 mag difference as the threshold, based on three factors: (i) given the QSO luminosity function (Willott et al. 2010; Ross et al. 2013; Shen et al. 2020), this corresponds to a factor of more than 10 in the number of objects, and should imply a large enough sample depth; (ii) optical variability is rare above $\sim 0.5 \mathrm{mag}$ amplitude, ensuring that the effective difference is not diminished substantially; and (iii) the pair at $z=5$ found by McGreer et al. (2016) has a similar $i^{\prime}$-band magnitude difference (19.4 versus $21.4 \mathrm{mag}$ ). Thus, exposure lengths of typically 20 minutes were decided upon, ensuring that the $3 \sigma$ limiting magnitude in the $z^{\prime}$ band was at least 2 mag deeper than the brightness of the spectroscopically identified QSOs (see below). While this 2 mag difference might suggest that the search could have been done just on Pan-STARRS data with its typical $5 \sigma$ depth of $z^{\prime}<22.3 \mathrm{mag}$, only 11 PS1 QSOs are bright enough to also survive the colour cut of $i^{\prime}-z^{\prime}>1.5 \mathrm{mag}$ at that faint $z^{\prime}$ brightness (see below). 


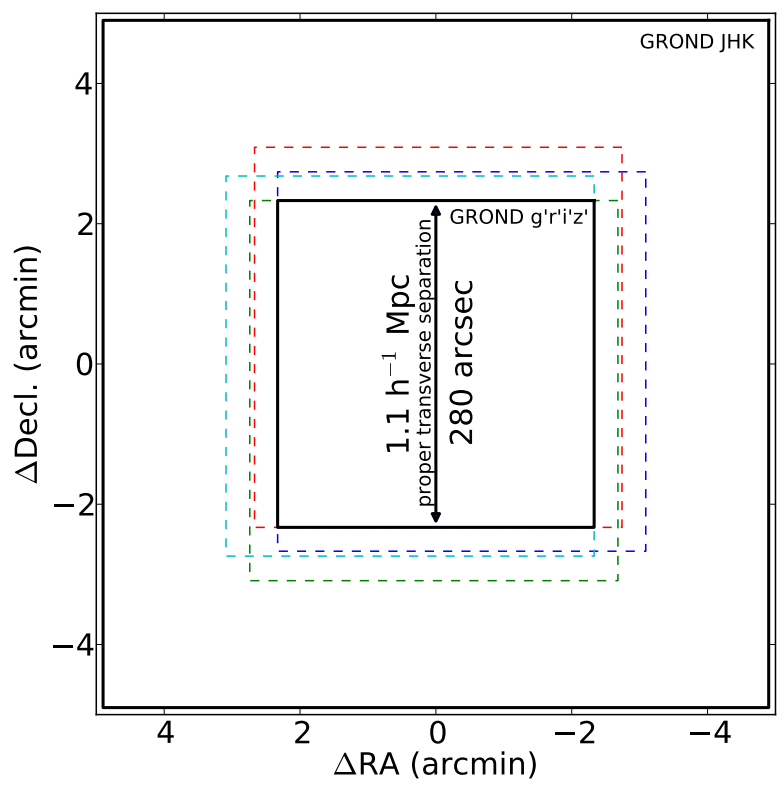

Fig. 1. Schematic of the GROND optical $\left(g^{\prime} r^{\prime} i^{\prime} z^{\prime}\right)$ and NIR (JHK) channel spatial coverage at a redshift of 6 . The dashed boxes are the respective $g^{\prime} r^{\prime} i^{\prime} z^{\prime}$ FOV for each individual telescope dither position; a similar pattern applies to the $J H K$ channels (not shown). With most of our targets being within 1 arcmin from the centre of the FOV, and since we require the $r^{\prime} i^{\prime} z^{\prime}$ bands, our conservative search radius is $80^{\prime \prime}$, corresponding to about $471 h^{-1} \mathrm{kpc}$ proper, or $3.3 h^{-1} \mathrm{Mpc}$ comoving.

GROND data were reduced in the standard manner (Krühler et al. 2008) using pyraf/IRAF (Tody 2019; Küpcü et al. 2008). The optical imaging data $\left(g^{\prime} r^{\prime} i^{\prime} z^{\prime}\right)$ was calibrated against the Sloan Digital Sky Survey (SDSS) ${ }^{1}$ (Eisenstein et al. 2011), Pan-STARRS $1^{2}$ (Chambers et al. 2016) or the SkyMapper Survey $^{3}$ (Wolf et al. 2018) catalogues, and the NIR data $\left(J H K_{\mathrm{s}}\right)$ against the 2MASS catalogue (Skrutskie et al. 2006). This results in typical absolute accuracies of $\pm 0.03 \mathrm{mag}$ in $g^{\prime} r^{\prime} i^{\prime} z^{\prime}$ and $\pm 0.05 \mathrm{mag}$ in $J H K_{\mathrm{s}}$. Since the GROND dichroics were built after the Sloan filter system (Greiner et al. 2008), the colour terms are very small, below $0.01 \mathrm{mag}$, except for the $i^{\prime}$ band which is substantially narrower than the SDSS i' band. All of our analysis was done in the SDSS system, and the following transformations were used: $g_{\text {SDSS }}^{\prime}-g_{\text {GROND }}^{\prime}=(-0.006 \pm$ $0.014)+(0.015 \pm 0.025) *\left(g_{\mathrm{SDSS}}^{\prime}-r_{\mathrm{SDSS}}^{\prime}\right), r_{\mathrm{SDSS}}^{\prime}-r_{\mathrm{GROND}}^{\prime}=$ $(-0.004 \pm 0.004)+(0.012 \pm 0.015) *\left(r_{\mathrm{SDSS}}^{\prime}-i_{\mathrm{SDSS}}^{\prime}\right), i_{\mathrm{SDSS}}^{\prime}-i_{\mathrm{GROND}}^{\prime}=$ $(-0.023 \pm 0.010)+(0.216 \pm 0.054) *\left(i_{\text {SDSS }}^{\prime}-z_{\text {SDSS }}^{\prime}\right), z_{\text {SDSS }}^{\prime}-$ $z_{\text {GROND }}^{\prime}=(-0.003 \pm 0.005)-(0.009 \pm 0.027) *\left(i_{\text {SDSS }}^{\prime}-z_{\text {SDSS }}^{\prime}\right)$. For the fields calibrated against Skymapper and PS1, we used their conversion to SDSS (Scolnic et al. 2015). The PS $1 z^{\prime}$ band has a substantial colour term $\left(z_{\text {GROND }}^{\prime}=z_{\text {PS1 }}^{\prime}-0.214 \times\left(z_{\text {PS1 }}^{\prime}-y_{\text {PS1 } 1}^{\prime}\right)\right.$ due to the missing tail beyond $920 \mathrm{~nm}$, but this becomes important only for redshifts above 6.6 when Lyman- $\alpha$ emission (Ly- $\alpha$ ) moves beyond the $z^{\prime}$-band limit.

The calibrated $z^{\prime}$ - and $J$-band images for each field were stacked after re-sampling due to their different pixel sizes $\left(0^{\prime \prime} \cdot 15\right.$ for the visual, and 0 '.59 for the NIR); for the four objects at $z>6.5$ we just used the $J$-band images. A source detection procedure on each stacked image then provided a list of all objects per field, which was then used as input for 'forced' photometry on the individual seven-band images. This provides upper lim-

\footnotetext{
http: //www.sdss3.org/

http://pan-starrs.ifa.hawaii.edu

http://skymapper.anu.edu.au
}

its for the bands where the sources are not detected; otherwise it performs point spread function (PSF) photometry for $g^{\prime} r^{\prime} i^{\prime} z^{\prime}$, and aperture photometry in $J H K$ (with a radius of $1 \times F W H M$ ) at the position of the source in the master catalogue which is allowed to re-centre by \pm 0.3 of the PSF width. Table A. 1 contains the details of the observation for each QSO, including the $3 \sigma$ limiting magnitude in the $z^{\prime}$-band. Table A.2 contains the foreground $A_{\mathrm{V}}$-corrected $\mathrm{AB}$ magnitudes of all $116 \mathrm{QSO}$ in the individual GROND filter bands.

\subsubsection{Colour-colour selection of candidates}

With these GROND data in hand, the goal was to search the field around each spectroscopically confirmed QSO for a nearby source with a similar colour, taking advantage of the simultaneous multi-filter optical-NIR spectral energy distributions (SEDs) of all objects inside the FOV.

We implicitly assume that the searches leading to the discovery of the QSOs pick the brighter of the pairs; this is corroborated by modelling which suggests that current flux-limited surveys of QSOs at high redshift preferentially detect objects at their peak luminosity, and thus miss a substantial population of similarly massive BHs accreting at lower accretion rates (Costa et al. 2014). Thus, here we are looking for objects fainter than the original QSO.

In all 116 fields we apply the following two colour selections (all in $\mathrm{AB}$ ): $i^{\prime}-z^{\prime}>1.5 \mathrm{mag}$, and $z^{\prime}-J>-0.7 \mathrm{mag}$. We checked that there are no detections in the $g^{\prime}$ and $r^{\prime}$ bands. For the $z>6.5$ objects, we adjust the $z^{\prime}-J$ colour to -0.5 , add $J-H<-0.5$, and require a $i^{\prime}$ non-detection. This returns 74 new high- $z$ quasar candidates. We excluded 48 candidates through visual inspection of the seven-channel image cut-outs of these candidates, based on either source confusion (i.e. a nearby source with overlapping PSF or an extended source that could be an interloper galaxy) or a non-resolved equally bright pair of objects. The remaining 26 candidate sources are shown in Fig. 2 as red dots (light and dark), overplotted on the distribution of the spectroscopically confirmed quasars (shown in blue), which by itself show that our colour cut criteria recover all but 13 objects, most of which fail due to non-detection (i.e. upper limits in the $i^{\prime}$-band, but deeper $i^{\prime}$-band exposure would likely recover those as well).

For 9 of the remaining 26 candidate sources we found catalogue entries in the ALLWISE all-sky catalogue (Wright et al. 2010; Mainzer et al. 2011) within 1 arcsec distance, and after a visual check of the images we accepted them as matching objects. Tables A.4 and A.5 contain the photometry of these 26 candidates, separately for those with and without ALLWISE matches.

We then performed Le PHARE (Arnouts et al. 1999; Ilbert et al. 2006) fits to the photometry of the 26 candidates (including the ALLWISE magnitudes when available). We included an alternative IGM opacity model (Songaila 2004) in addition to the default one (Madau et al. 1999), but otherwise used the default spectral templates except for adding about 200 cloned high- $z$ QSO templates based on stacked observed SDSS spectra of low- $z$ QSOs, similar to Willott et al. (2007a). The goal of these Le PHARE fits was to distinguish late-type dwarfs from QSO candidates, where the distinction is based on two observational features: the steepness of the SED in the $i^{\prime}-z^{\prime}-J$ range, and the SED shape in the $H K$ range which is much redder for dwarfs. Since dwarfs in our sensitivity range would be at distances of order $100 \mathrm{pc}$, their photometry would not be affected by interstellar dust. We thus created two SEDs for each candidate, one without $A_{\mathrm{V}}$-correction (for Le PHARE fits with dwarf 

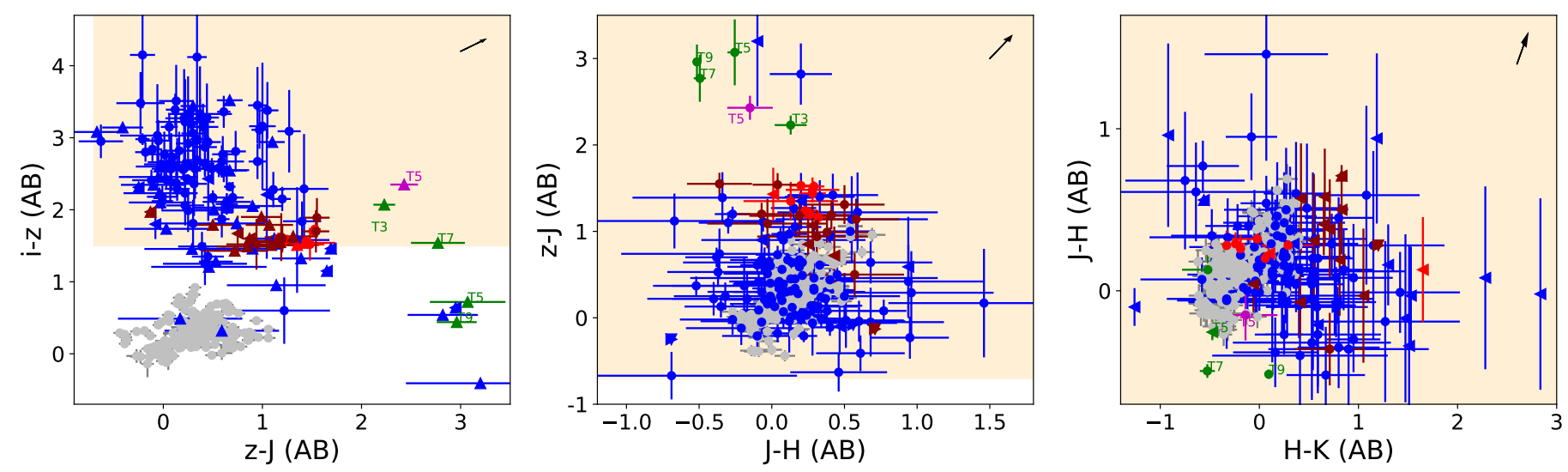

Fig. 2. Colour-colour diagram in the GROND-intrinsic $i^{\prime} z^{\prime} J H K_{s}$ bands of the prime quasars (in blue; triangles denote limits) compared to normal foreground stars and galaxies (in grey) of the same fields, with selected brown dwarfs from earlier GROND observations (in green and purple, and labelled with their spectral types). Our 26 candidates are shown in red; the nine objects which have ALLWISE detections are in light red. The yellow-shaded region indicates the search region for the QSO pair. The arrow at the top right of each panel shows the amount by which a correction for $A_{\mathrm{V}}=1$ would shift an object. Except for the grey objects, all are foreground $A_{\mathrm{V}}$-corrected.

templates) and one with full galactic $A_{\mathrm{V}}$-correction (for Le PHARE fits with QSO templates). For 14 of the 26 candidates the best-fit template is a dwarf (see last column of Tables A.4 and A.5), for 10 candidates a QSO at high redshift (labelled in boldface in Table A.5), for 1 candidate a galaxy at intermediate redshift, and for 1 candidate (SDSSJ2054-0005_4) the reduced $\chi^{2}$ does not provide a clear favorite. The Le PHARE fits of the ten high- $z$ quasar candidates (i.e. with $A_{\mathrm{V}}$-correction applied) are shown in Fig. 6, which also contains the best-fit photo- $z$ (sixth field in the red line of labels), all within $13 \%$ of the spectroscopic redshift except for PSO J055.4244-00.8035 (the source whose spectrum we did not obtain).

\subsection{Spectroscopic observations}

We obtained spectroscopy of 11 objects (9 of the 10 QSO candidates, the galaxy, and the unidentified object), mostly with the GMOS instruments of the Gemini Observatory (under proposal IDs GN-2019B-FT-201 and GS-2019B-FT-202). One object was observed with the Folded-port InfraRed Echellette (FIRE) spectrograph in Prism mode at the Magellan Baade telescope. A $\log$ of the observations is given in Table A.3. At Gemini-North (South), a Hamamatsu detector was used with the R150 grating and filter G5308 (G5326). The spectra cover the range from 615 (750) to $1080 \mathrm{~nm}$. Wavelength calibration was provided by $\mathrm{CuAr}$ comparison lamps. Flux calibration was done against the standard stars G191B2B (LTT7987). For the FIRE spectrum, we used archival A0V stars for telluric and flux calibration. The data were reduced in the standard manner, using GMOS specific routines provided within the IRAF package (Tody 2019), and a custom-made python code for the FIRE observation.

For object PSOJ071.0322-04.5591 no trace is visible in the 2D spectrum; for the other sources the optimally extracted 1D spectra are shown in Fig. 7. None shows a sign of the Ly- $\alpha$ 'step' at the wavelengths suggested by the photometric redshifts (vertical lines in Fig. 7).

\section{Results and discussion}

\subsection{Pair candidates}

We do not find a single QSO pair candidate brighter than $M_{1450}(\mathrm{AB})<-26 \mathrm{mag}$ in our GROND data of 116 spectroscopically confirmed redshift 6 quasars. Except for two candidate objects (near PSOJ055.4244-00.8035 and PSOJ071.0322-04.5591) we obtained optical spectroscopy for all the other Le PHARE-derived QSO pair candidates, but could not confirm the quasar nature suggested by the colour selection and SED fitting. This is somewhat surprising, since the (ex post facto) photometric redshifts we derived with Le PHARE for the prime QSOs where in good agreement with the spectroscopic redshifts reported by Bañados et al. (2016), with only a $5 \%$ fraction mismatch. We consider it very unlikely that there is an unrecognised quasar among our identified candidates in Tables A.4 and A.5. This is in contrast to our empirical expectation (at the start of the project) of three pairs in our sample, based on the serendipitously discovered pair at $z=4.26$ (Schneider et al. 2000) and the two pairs at $z=5.02$ (Djorgovski et al. 2003; McGreer et al. 2016) (see details in the introduction), which when averaged together (1/100, 1/14, 1/47) suggest one pair every 40 QSOs, or $2.5 \%$. This line of reasoning includes a few simplifications. First, it uses ex post facto arguments and guesses on the search volume of the above serendipitous discoveries. Next, it assumed that the quasar clustering fraction does not evolve with redshift, the question to be answered by the project. It also ignored the different luminosity ranges sampled at different redshifts; the integral over the luminosity function is certainly the most sensitive component contributing to the $w_{\mathrm{p}}$ statistic (see below).

In order to put this in context, we follow Hennawi et al. (2006) and Shen et al. (2010, in particular their Eq. (4)) in estimating the projected correlation function, or $w_{\mathrm{p}}$ statistics, as defined by Davis \& Peebles (1983), among others. Given the mean seeing in our images and correspondingly the ability to separate two sources, we take an inner radius of $100 \mathrm{~h}^{-1} \mathrm{kpc}$ (corresponding to $3^{\prime \prime}$ ) for the projected comoving area of the cylindrical annulus (the outer one being our search radius of $3.3 h^{-1} \mathrm{Mpc}$ ). We use the latest luminosity function description of Kulkarni et al. (2019), as well as their publicly provided tools ${ }^{4}$ to convert our observed $z^{\prime}(\mathrm{AB})$ magnitude limit and mean redshift to $M_{1450}$, and applying the corresponding bolometric correction (using their 'global fit A'; we verified our procedure against their Fig. 5). This results in a number density of $9 \times 10^{-10} \mathrm{Mpc}^{-3}$ for quasars with $M_{1450}<-26 \mathrm{mag}$, for our

\footnotetext{
4 https://github.com/gkulkarni/QLF
} 


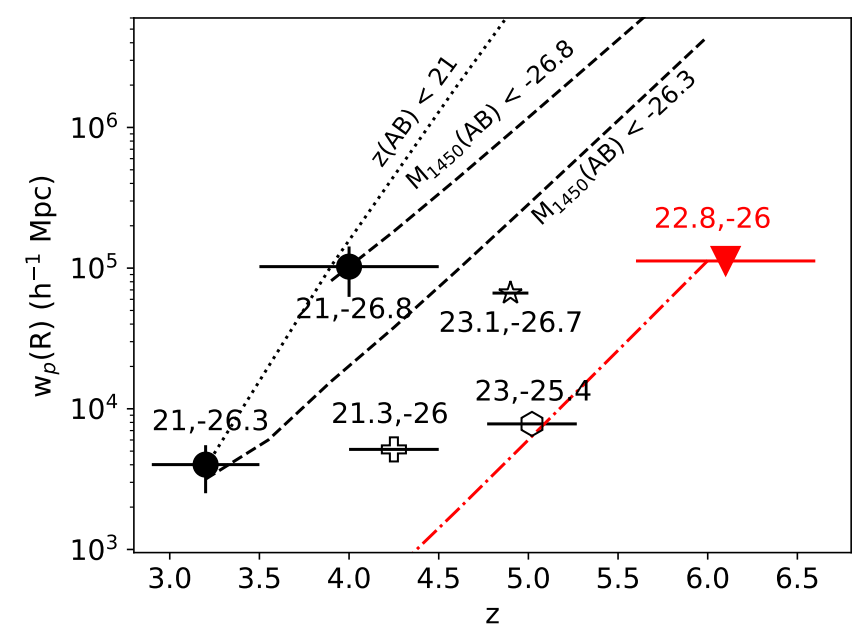

Fig. 3. Our upper limit (red triangle) on the projected correlation function of $z \sim 6$ QSOs, as derived from the GROND observations. The two filled circles are the corresponding findings by Shen et al. (2010) for 7 and 8 pairs, respectively, modified for the different luminosity function (and cosmology) used over time, and adapted to our search scale of $0.1-3.3 h^{-1} \mathrm{Mpc}$ according to the -2 power-law scale dependence. Open symbols indicate the serendipitous discoveries from Schneider et al (2000), Djorgovski et al. (2003), and McGreer et al. (2016), also extrapolated to our search scale. The horizontal error bars show the corresponding redshift range of the studies. The labels at the data points report the limiting $i$-/z-band $\mathrm{AB}$ magnitude of the search (see Col. 5 of Table 1) and the corresponding absolute $M_{1450}(\mathrm{AB})$ limiting magnitude. The two black dashed lines depict $w_{\mathrm{p}}(\mathrm{R})$ for the same rate of 7 and 8 pairs, as in Shen et al. (2010), extrapolated to higher redshift with their same $M_{1450}(\mathrm{AB})$, and unchanged luminosity function, suggesting higher $w_{\mathrm{p}}(\mathrm{R})$ at higher redshift. Similarly, the dotted line shows the extrapolation for the case of constant limiting $z$-band magnitude. The red line depicts the backwards extrapolation of our upper limit to lower redshifts, at our $M_{1450}(\mathrm{AB})<-26$ mag sensitivity, demonstrating the mismatch with respect to the findings at lower redshift. All the lines are computed via integration over the luminosity function of Shen et al. (2020) in redshift steps of 0.25 , and proper redshift-dependent transformation between observed $z^{\prime}$ and absolute magnitudes.

redshift range $5.6<z<6.6$. We obtain an upper limit of $w_{\mathrm{p}}<110000 h^{-1} \mathrm{Mpc}$ (see Fig. 3), where we use a formal upper limit of our search of one pair out of the 116 objects searched for, given the missing spectroscopy for PSOJ055.4244-00.8035. The figure also includes measurements at lower redshifts from Schneider et al. (2000), Shen et al. (2010), and McGreer et al. (2016) (modified by the updated luminosity function and corrected for our search scale according to the $\gamma=2$ power-law dependence of $w_{\mathrm{p}}(R) \propto R^{-\gamma}$ from Shen et al. 2010). Overall, our upper limit corresponds to a similar $w_{\mathrm{p}}$ as previous findings at lower redshift, suggesting that there is no strong rise of clustering towards high redshift.

Using Eq. (5) in Shen et al. (2007) and $\gamma=2$, our upper limit on $w_{\mathrm{p}}$ corresponds to a limit of the auto-correlation length $r_{o}<1000 h^{-1} \mathrm{Mpc}$.

\subsection{Completeness and biases}

In the past clustering studies have typically relied on selecting pairs from large catalogues (e.g., SDSS), and the most important question is about the completeness of the underlying catalogue (e.g., Hennawi et al. 2006; Eftekharzadeh et al. 2017). Here, we obtained follow-up observations of known quasars at known redshifts. Thus, in our case the question of completeness reduces to the questions of (i) whether or not the selection criteria which led to the sample of these known redshift quasars had a bias against selecting binary quasars and (ii) whether our search strategy misses QSOs. As to item (i), one potential bias is that the seeing limit in the original survey data affects the discovery of QSO on 1-2" scales due to nearby contaminating sources. As this is a statistical problem, it is not expected to affect the fraction of binary QSOs in the spectroscopically confirmed QSO sample.

As to item (ii), this splits into two sub-questions. First, what is the completeness of our colour-colour cuts? Since we ran our colour-colour selection blindly on all sources in each observation, we can answer this with the recovery rate of the spectroscopically confirmed QSO: out of the 116 QSOs, we missed 9: 3 not detected in $z^{\prime}$, and the other 6 due to insufficiently deep $i^{\prime}$ band limits. Since the three non-detections are due to variability, they are not counted here (see below on the variability bias), so our incompleteness of the colour-colour selection is about $5 \%$. Second, what is the completeness of the Le PHARE fits in recognizing the QSO nature? Again, this can be answered with the sample of the spectroscopically confirmed QSOs. Ignoring sources with detections in fewer than three photometric bands, we ran Le PHARE fits on the remaining 97 spectroscopically confirmed QSOs in the same manner as with the candidates (i.e. two runs, one with and one without $A_{V}$ correction). Only 5 sources are classified as dwarfs, the other 92 as QSOs. We double-checked that the colours and photometric errors of the 5 sources are not outliers with respect to the sample distribution of the 92 correctly classified QSOs. So this corresponds to only $<5 \%$ incompleteness.

Further, we note that it is unlikely that even substantial optical variability of the QSOs would affect the fraction of pairs, since statistically the same number of 'risers' versus 'faders' is expected. The only exception would be effects due to Malmquist bias, but since only a handful of objects are detected with a $z^{\prime}$-band uncertainty larger than $0.2 \mathrm{mag}$, this imbalance is unimportant.

Lastly, a potential bias could be due to dust obscuration of AGN, in our case obscuring the companion of the spectroscopically confirmed QSO. Again, this is not expected to be a major issue. First, previous suggestions were that obscuration could primarily happen in the highest mass accretion phases; one would first expect the brighter object of the pair to be obscured, rather than the lower-luminosity companions of the unobscured spectroscopicall confirmed QSOs. Second, while ALMA observations revealed substantial dust at high redshift, there is no evidence that these dusty objects are obscured AGN (see Sect. 3.4 below). Finally, theory of dust production fails to explain dust in large amounts at $z>5$ (Leśniewska \& Michalowski 2019), preventing quantitative estimates or simulations.

Another potential bias could come in if BHs of similar mass have different Eddington ratios at different redshifts, such that only the optically brightest QSOs are found at high redshift. However, Mazzucchelli et al. (2017b) show that (at least for the biased high- $z$ sample they considered) BH masses and Eddington ratios of the $z>6.5$ and SDSS $0.35<z<2.25$ samples are consistent in a luminosity-matched sample. Thus, our assumption was that the clustering fraction at redshift 4-5 (Schneider et al. 2000; McGreer et al. 2016) is not much different than at redshift 6 , leading to our expectation of more pairs compared to our findings.

Furthermore, our search comes with the biases that the luminosity difference between the two members of the pair can be larger than our selected $2 \mathrm{mag}$, for at least two different reasons. Firstly, the BH masses and/or accretion rates can differ 
Table 1. Comparison of search volumes and depth for previous, serendipituously found high-z QSO pairs.

\begin{tabular}{lccccc}
\hline \hline Publication & No. of pairs & z-range & $\begin{array}{c}\text { Separation } \\
(\operatorname{arcsec}) /(\mathrm{cMpc})\end{array}$ & $\begin{array}{c}\text { Depth } \\
(\mathrm{AB} \mathrm{mag})\end{array}$ & Comments \\
\hline Shen et al. (2010) & 7 & $2.9-3.5$ & $-/ 1.0$ & $i<21.0$ & 1 \\
& 8 & $3.5-4.5$ & $-/ 1.2$ & $i<21.0$ & 1 \\
Schneider et al. (2000) & 1 & $4.0-4.5$ & $33 / 1.2$ & $i<21.0$ & 2 \\
McGreer et al. (2016) & 1 & $4.77-5.27$ & $21 / 0.8$ & $z<21.4$ & 3 \\
Djorgovski et al. (2003) & 1 & $4.8-5.0$ & $160 / 6.2$ & $z<23.1$ & 4 \\
This paper & 0 & $5.6-6.6$ & $3-80 / 3.3$ & $z<22.8$ & \\
\hline
\end{tabular}

Notes. ${ }^{(1)}$ Out of 319 binary candidates, follow-up spectroscopy 'is about half finished' (Hennawi et al. 2010). Based on the spectroscopic followup, Shen et al. (2010) derive a search completeness of 0.38 and 0.52 for the low- $z$ and high- $z$ sub-sample, respectively. These completeness fractions are used in computing $w_{\mathrm{p}}$ for Fig. 3 . $^{(2)}$ The second QSO of the pair was serendipitously in the slit, so we use the pair separation of 33" even though it does not correspond to an azimuthally complete search cone. ${ }^{(3)}$ The second QSO of the pair was serendipitously in the slit, so we use the pair separation of $21^{\prime \prime} .{ }^{(4)}$ The redshift depth is taken as the highest redshift QSO found in the parent sample. The search radius is estimated from the imaging FOV and guessing a 1 arcmin loss in the side-length due to dithering.

substantially, and secondly the Ly- $\alpha$ emission, which contributes to the $z$-band magnitude, can be drastically different between the two members of the pair, replicating the differences in the spectroscopically confirmed QSO sample (see e.g., Bañados et al. 2016). Regarding the first reason, simulations suggest differently. Based on the large-scale cosmological hydrodynamical Horizon-AGN simulations, Volonteri et al. (2016) find that the luminosity ratio between the central $\mathrm{BH}$ (the more massive one) and an off-centre $\mathrm{BH}$ is within a factor of ten $(\mathrm{rms}=5 \mathrm{x})$ for the cases of $L_{\text {bol }}>10^{43} \mathrm{erg} \mathrm{s}^{-1}$ and BH masses up to $10^{8} M_{\odot}$. While this corresponds to the ratio we employed, it should be noted that this ratio depends on selected input parameters for the simulation, among others a mass ratio $<1: 6$ for the merging galaxies at which AGN activity is triggered in both BHs. Similarly, Bhowmick et al. (2019) find that in the MassiveBlack II simulations the satellite quasar luminosity is similar to that of central quasars. Finally, we note that for 41 of our targets the $3 \sigma$ limiting $z^{\prime}$ magnitude reached in our observations is actually 3 mag fainter than that of the spectroscopically confirmed QSO, which still would lead to an expectation of one pair in our sample.

\subsection{Comparison to simulations}

The upper limit to the projected correlation function for bright QSO pairs at $z \sim 6$ presented in Sect. 3.1 serves as an important constraint for galaxy evolution models. Currently, cosmologicalscale simulations typically either focus on quasar properties below $z \sim 5$ due to improved statistics (e.g., DeGraf \& Sijacki 2017; Bhowmick et al. 2019; Habouzit et al. 2021) or above $z \sim$ 7 due to reduced computational limitations (e.g., Di Matteo et al. 2017; Tenneti et al. 2019; Marshall et al. 2020). Zoom-in simulations of halos containing QSOs at $z \sim 6-7$ have been performed (e.g., Costa et al. 2014; Lupi et al. 2019); however, they do not readily provide statistics on large scales. Consequently, model predictions for the total number of bright QSO pairs at the redshift of our sample are still lacking in the literature.

At lower redshifts models suggest that bright QSO pairs are rare. For example, in the MASSIVEBLACK-II simulation box of volume $\left(100 h^{-1} \mathrm{Mpc}\right)^{3}$, Bhowmick et al. (2019) find no pairs of QSOs with $g<20.85$ within a search radius of $4 \mathrm{Mpc}$ at $z=2$, and only three such systems by $z=0.6$.

At higher redshift, work on the BLUETIDEs simulation has shown that QSOs bright enough to match observations are also very rare, even when accounting for large $\mathrm{BH}$ seeds (Di Matteo et al. 2017). There is only one BH of mass above
$10^{8} M_{\odot}$ present in the BLUETIDES $\left(400 h^{-1} \mathrm{Mpc}\right)^{3}$ box at $z=$ 8 , which has a luminosity of $L_{\text {bol }} \sim 2.2 \times 10^{46} \mathrm{erg} \mathrm{s}^{-1}$ (Di Matteo et al. 2017; Tenneti et al. 2019).

However, despite the lack of pairs, galaxy evolution models still find that brighter QSOs cluster more strongly than their fainter counterparts, particularly on smaller scales. For example, on scales of $\sim 10 \mathrm{kpc}$ at $z=4$, DeGraf \& Sijacki (2017) find the two-point auto-correlation function, $\xi(r)$, for AGN with $L_{\text {bol }} \geq 10^{44} \mathrm{erg} \mathrm{s}^{-1}$ in the ILLUSTRIS simulation is higher by a factor of three compared to that for all AGN. On the larger scales probed by our observational measurement $\left(\sim 0.1-3.3 h^{-1} \mathrm{Mpc}\right)$ this enhancement is reduced, but still remains present for the brightest objects, as also seen in the L-GALAXIES semi-analytic model (Bonoli et al. 2009) and the HoRIZON-AGN simulation (Volonteri et al. 2016). All three of these models report a value of $\xi\left(r=1.2 h^{-1} \mathrm{Mpc}\right) \sim 10$ at $z=4$ for AGN with $L_{\mathrm{bol}} \geq$ $10^{44} \mathrm{erg} \mathrm{s}^{-1}$, with the expectation that this value will be higher at $z=6$, as also seen in the observations discussed in Sect. 1 .

In order to better probe the exact epoch and search volume of our observational QSO sample, we carried out a census of bright AGN pairs at $z=6$ in the ILLUSTRIS-TNG300 (hereafter TNG300) and L-GALAXIES 2020 galaxy evolution models. TNG300 (Pillepich et al. 2018; Springel et al. 2018; Naiman et al. 2018; Marinacci et al. 2018; Nelson et al. 2018) is a magneto-hydrodynamical simulation run in a $\left(205 \mathrm{~h}^{-1} \mathrm{Mpc}\right)^{3}$ box, using a Bondi-Hoyle-Lyttleton $\mathrm{BH}$ accretion model with no boost factor but an assumed BH seed mass of $8 \times 10^{5} M_{\odot}$ (see Habouzit et al. 2021). L-GALAXIES 2020 (Henriques et al. $2020)$ is a semi-analytic model run on the larger $\left(480 h^{-1} \mathrm{Mpc}\right)^{3}$ MiLLENNIUM-I box (Springel et al. 2005), but with a more simplified phenomenological model of quasar- and radio-mode $\mathrm{BH}$ accretion and no BH seed mass (see Croton et al. 2006). In both cases we assume that all AGN are radiatively efficient when calculating their luminosities, which provides an upper limit on the expected number of bright pairs. Therefore, the AGN bolometric luminosity for both models is calculated here as

$L_{\mathrm{bol}}=\frac{\epsilon_{\mathrm{r}}}{1-\epsilon_{\mathrm{r}}} \dot{\mathrm{M}}_{\mathrm{BH}} c^{2}$,

where $\epsilon_{\mathrm{r}}$ is the assumed radiative efficiency of the accretion disc, $\dot{\mathrm{M}}_{\mathrm{BH}}$ is the $\mathrm{BH}$ accretion rate, and $c$ is the speed of light. Given that the detection limit for the fainter of the two QSOs in our observational search is $M_{\mathrm{B}} \sim-26 \mathrm{mag}$ (i.e., $L_{\mathrm{B}} \sim 8.25 \times$ $10^{45} \mathrm{erg} \mathrm{s}^{-1}$ ), we assume a minimum bolometric luminosity of $L_{\text {bol,min }}=4 \times 10^{46} \mathrm{erg} \mathrm{s}^{-1}$ when searching for BH pairs in the 


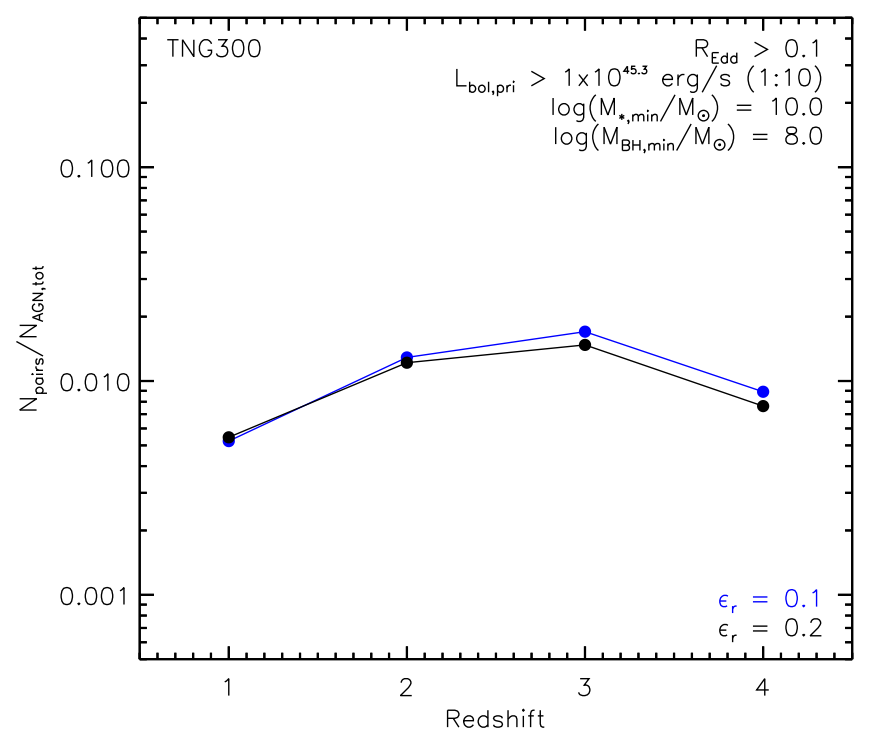

Fig. 4. Fraction of BH pairs over redshift in TNG300, for two values of $\epsilon_{\mathrm{r}}$. Following Silverman et al. (2020), pairs are selected to have primary BH luminosities of $L_{\text {bol,pri }} \geq 10^{45.3} \mathrm{erg} \mathrm{s}^{-1}$, secondary BH luminosities of $L_{\mathrm{bol}, \text { sec }} \geq L_{\mathrm{bol}, \text { pri }} / 10$, a minimum BH mass of $10^{8} M_{\odot}$, and a minimum host-galaxy stellar mass of $10^{10} M_{\odot}$. At redshifts $>5$ there are AGN, but no pairs.

models, given the bolometric conversion factor of 5.13 recommended by Duras et al. (2020).

For the same search area as our observational sample (i.e., an inner radius of $125 h^{-1} \mathrm{kpc}$ and outer radius of $3.3 h^{-1} \mathrm{Mpc}$ comoving, see Sect. 3.1), we find no BH pairs with $L_{\text {bol }} \geq$ $4 \times 10^{46} \mathrm{erg} \mathrm{s}^{-1}$ in either TNG300 or L-GALAXIES 2020 at $z=6$. This holds true even when doubling the assumed radiative efficiency from $\epsilon_{\mathrm{r}}=0.1$ to 0.2 . There are no BHs at all at that luminosity within the TNG300 box above $z=5$ when assuming $\epsilon_{\mathrm{r}}=0.2$. This theoretical result is nicely consistent with the null result returned by our observational search at $z=6$, especially when considering the relatively large box sizes of L-GALAXIES 2020 and TNG300.

We do not find any BH pairs at $z=5$ in TNG300 or L-GALAXIES 2020 that match the serendipitous discovery by McGreer et al. (2016) of a bright pair with $M_{1450} \leq-25.4$ (i.e., $L_{\text {bol }} \geq 2.4 \times 10^{46} \mathrm{erg} \mathrm{s}^{-1}$ ) separated by $21^{\prime \prime}$ (i.e., $816 \mathrm{ckpc}$ ) at $z=5.02$. These simulations are therefore somewhat in tension with this discovery, although the exact search area to use is not well constrained. However, we do find pairs in TNG300 that match the serendipitous discovery on a BH pair with $M_{\mathrm{B}} \leq$ -23.7 mag (i.e., $L_{\text {bol }} \geq 5.1 \times 10^{45} \mathrm{erg} \mathrm{s}^{-1}$ ) by Schneider et al. (2000) at $z=4.26$. When assuming $\epsilon_{\mathrm{r}}=0.1$, and a standard search volume around the mean deprojected separation of $r=1.92 \mathrm{cMpc}$ with inner radius $r-(r / 4)=1.44 \mathrm{cMpc}$ and outer radius $r+(r / 4)=2.40 \mathrm{cMpc}$, we find $14 \mathrm{BH}$ pairs with $L_{\text {bol }} \geq 5.1 \times 10^{45} \mathrm{erg} \mathrm{s}^{-1}$ at $z=4$ in TNG300. This equates to an upper limit on the pair fraction (i.e., the number of $\mathrm{BH}$ pairs divided by the total number of BHs at that luminosity) of $\sim 3.1 \%$. We are also able to reproduce a fairly redshift-invariant pair fraction of $\sim 1 \%$ between $z \sim 4$ and 1 (see Fig. 4) for BH pairs with a primary BH luminosity of $L_{\text {bol,pri }} \geq 1 \times 10^{45.3} \mathrm{erg} \mathrm{s}^{-1}$, when selecting systems as Silverman et al. (2020) do for the HoRIZON-AGN simulation (see their Sect. 5.1). No such pairs were found in TNG300 above $z=4$.

In conclusion, the observed number of bright QSO pairs seen at high redshift appears largely consistent with that predicted by current cosmological-sized galaxy evolution models, although further modelling efforts at the redshift of our observational sample are still required to confirm this.

\subsection{Number of QSO pairs as a constraint on massive $B H$ origins and assembly}

The origin of $M_{\mathrm{BH}} \sim 10^{8-9} M_{\odot}$ BHs in massive galaxies at $z \sim 6-7$ is largely unconstrained (e.g., Johnson et al. 2013; Valiante et al. 2017, 2018; Inayoshi et al. 2020). The cosmic time since the Big Bang is likely too short for light seeds with masses of $\sim 100 M_{\odot}$ (e.g., the PopIII remnant model, Madau \& Rees 2001; Bromm \& Loeb 2003; Volonteri et al. 2003b) to grow to such masses even if the seeds accrete at the Eddington rate most of their lifetimes. Instead, more massive seeds with masses of order $\sim 10^{4} M_{\odot}$ would have a somewhat less challenging growth history to reach the masses of the high- $z$ QSOs.

Several theoretical models have been put forward to explain the formation of such massive $\mathrm{BH}$ seeds. By analogy with the nuclear clusters that we commonly observe in low-redshift galaxies, compact nuclear clusters in metal-poor environments are predicted to form very massive stars by runaway stellar collisions (Omukai et al. 2008; Devecchi \& Volonteri 2009; Regan \& Haehnelt 2009). These very massive stars would collapse onto $\mathrm{BH}$ seeds of $M_{\mathrm{BH}} \sim 10^{2}-10^{3} M_{\odot}$. Seeds of about the same mass can be formed by runaway $\mathrm{BH}$ mergers in metal-poor star clusters in the centre of high-redshift galaxies. The direct collapse model predicts the most massive seeds, called direct-collapse BHs (DCBHs): atomic cooling halos form single supermassive stars, which can collapse onto massive seeds of $M_{\mathrm{BH}} \sim 10^{4}-10^{6} M_{\odot}$ (Loeb \& Rasio 1994; Bromm \& Loeb 2003; Spaans \& Silk 2006; Begelman et al. 2006; Lodato \& Narayan 2006; Dijkstra et al. 2008; Visbal et al. 2014; Latif \& Volonteri 2015; Habouzit et al. 2016b). In order to avoid the fragmentation of the gas into multiple and less massive stars, the presence or formation of efficient gas coolants (i.e. molecular hydrogen and metals) must be prevented. Therefore, the formation of the first DCBHs depends on the number of surrounding halos because the Lyman-Werner radiation (photons with energy in the $11.2-13.6 \mathrm{eV}$ range) needed to prevent $\mathrm{H} 2$ formation comes from the surrounding star-forming galaxies (Habouzit et al. 2016a).

These mechanisms yield different galaxy occupation fractions (i.e. the probability for a galaxy to host a $\mathrm{BH}$ ), and therefore could lead a priori to different predictions on the number of QSO pairs at high redshift. While the compact nuclear stellar cluster model and the model of runaway BH mergers in clusters predict potentially large occupation fractions (i.e. the presence of BHs in many galaxies), the number density of direct collapse BHs is predicted to be small (e.g., Dijkstra et al. 2008; Habouzit et al. 2016b). However, DCBHs could appear clustered. Whether the large Lyman-Werner radiation is produced by several or a single star-forming halo, the high intensity required could irradiate several nearby halos, potentially forming DCBHs in some of these halos if metal-poor conditions are met. The need for clustered halo environments is not required for all the variations of the direct collapse model (e.g., the synchronised pair model, Visbal et al. 2014). It has also been proposed that DCBHs themselves could ignite a runaway process of further DCBH formation (Yue et al. 2014). DCBHs are expected to be Compton thick, and their reprocessed radiation outshines small high-redshift galaxies by more than a factor of 10 . Thus, once the first DCBHs form, their Lyman-Werner radiation could help to 
provide surrounding halos with the needed conditions to form new DCBHs (Yue et al. 2014). This process comes to an end when the host atomic-cooling halos are rapidly photo-evaporated by ionizing photons. An analytical model of these processes (Yue et al. 2014) shows that a highly clustered spatial distribution of halos is the pre-requisite to triggering the formation of several DCBHs in the same region.

The different mechanisms of $\mathrm{BH}$ formation are not a priori mutually exclusive in the Universe, which therefore makes the interpretation of the QSO clustering difficult. Moreover, successive mergers of halos with time alter the initial halo occupation fraction and clustering of BHs at birth.

Observing several QSO pairs in our sample would likely suggest that massive $\mathrm{BH}$ seeds can form in the same regions (on scales of up to $1 \mathrm{Mpc}$ ), and that they can grow efficiently in the dense environments in which we often find high-redshift QSOs. The fact that we find no QSO pairs (i.e. no bright companion to any of our 116 spectroscopically confirmed QSOs) could mean that (i) most QSOs are isolated objects with no massive galaxy in their surroundings; (ii) QSOs are often located in dense environments, but nearby galaxies are devoid of BHs or devoid of massive BHs; or (iii) QSOs are often located in dense environments, and nearby galaxies also host massive $\mathrm{BHs}$; however, these $\mathrm{BHs}$ have high variability and/or low duty cycle and no companion BHs were active when observed.

While the question of whether QSOs are embedded in the densest environments is still subject to debate in observations (Kim et al. 2009; Husband et al. 2013; Mazzucchelli et al. 2017a; McGreer et al. 2016; Habouzit et al. 2019, and references therein) hypothesis (i) where a large fraction of QSOs are isolated objects is unlikely. Theoretical models suggest that the first massive galaxies formed through mergers of gas-rich galaxies at very high redshift. Recently, Decarli et al. (2017) and Neeleman et al. (2019) identified six $z>6$ QSOs with close gas-rich companions through ALMA observations of [CII] and dust emission; five of these sources are in our sample: SDSSJ0842+1218, PSOJ167.6415-13.4960, SDSSJ1306+ 0356, PSOJ308.0416-21.2339, and CFHQSJ2100-1715. These QSO-galaxy pairs prove that $z>6$ QSOs are not living isolated from the surrounding matter in their halos. This QSOgalaxy clustering is consistent with the quasar/LBG clustering at $z \sim 4$ (see Fig. 3 in Decarli et al. 2017). Recent deep X-ray observations of some of the above sources did not reveal potentially obscured quasars. To date, there is no strong evidence that these obscured companions of $z \sim 6$ quasars are obscured AGN (Connor et al. 2020; Vito et al. 2021).

Therefore, our non-detection of bright QSO-QSO pairs could hint towards an evolutionary scenario that prevents the formation of a massive accreting $\mathrm{BH}$ in the potentially gas-rich QSO companion. This could be due to tidal interaction of the primary QSO being more rapid than the accretion on the central $\mathrm{BH}$ of the companion. Feedback from the QSO could also reduce the gas reservoir of the companion galaxy through its lifetime, therefore diminishing the ability of a $\mathrm{BH}$ to efficiently accrete and become as massive as the $\mathrm{BH}$ powering the QSO. Additionally, efficient stripping of gas from companion galaxies of massive systems is seen out to several virial radii at low redshift in cosmological simulations (Ayromlou et al. 2021a,b). If this effect is present in the neighbourhood of the brightest QSOs at high redshift, it could also contribute to a reduction in the gas reservoir of companion BHs. The absence of QSO pairs could also be explained in case of low efficiency of $\mathrm{BH}$ formation, so that early massive $\mathrm{BH}$ formation happens only in a small fraction of galaxies, or alternatively if the formation of light seeds is

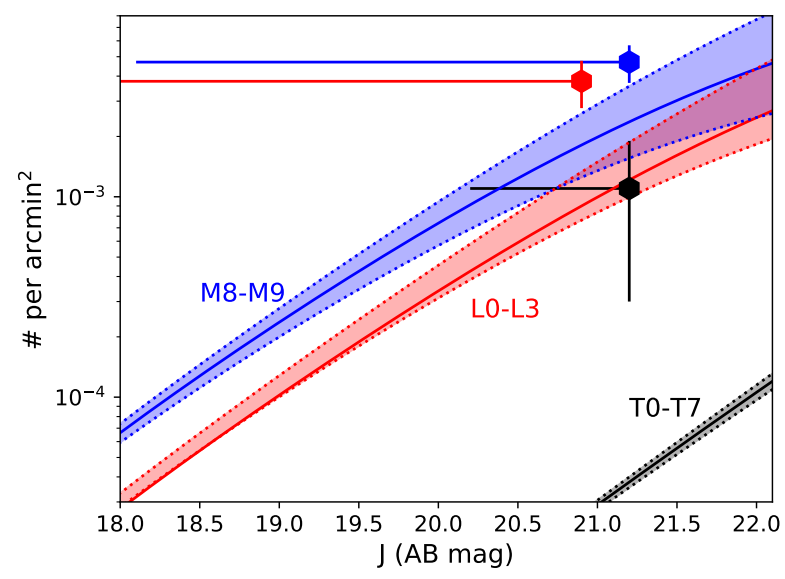

Fig. 5. Number of brown dwarfs among our sample of 26 candidates per total area of our search region over 116 QSOs, compared to the cumulative surface density as modelled by Ryan \& Reid (2016), plotted here for their COSMOS sample field (central line) as well as the fields with the smallest and largest numbers (corresponding to different galactic latitudes) providing the covered range (shaded area). Our data points are plotted at the faintest magnitude per spectral type range, and the horizontal bar covers the magnitude range up to the brightest. While the observed number of M dwarfs is compatible with the known surface density, that of $\mathrm{T}$ dwarfs is substantially larger.

dominant in the Universe. Due to their low masses, light seeds would have a hard time growing and even sinking efficiently to the potential well of their host galaxies. Searching for companions of the ten most massive BHs in TNG300 reveals none with masses $M_{\mathrm{BH}} \geq 10^{7.7} M_{\odot}$ at $z=5$ and above. There is just one companion BH with a mass of $M_{\mathrm{B} H} \sim 10^{7.3} M_{\odot}$ at $z=6$, but with a luminosity of only $L_{\mathrm{bol}}=10^{43.3} \mathrm{erg} \mathrm{s}^{-1}$, which is not even the brightest luminosity in the companion sample (which is $10^{45.1} \mathrm{erg} \mathrm{s}^{-1}$ ), and below our search sensitivity. With its limited volume of $\left(205 h^{-1} \mathrm{cMpc}\right)^{3}$ there is no environment in TNG300 that was able to build up two extremely massive and luminous BHs in the same region by $z \sim 6$.

Finally, this leads us to hypothesis (iii) where very short coincident activity periods of both QSOs (see e.g., Haiman \& Hui 2001 for an early account of the lifetimedependence of QSO clustering). In this case QSO companion galaxies would also host massive $\mathrm{BHs}$, but these $\mathrm{BHs}$ would not be efficiently accreting at the time of observation. In this case, the companion massive BHs would have grown by several orders of magnitude, potentially releasing a significant amount of energy in their host galaxies through cosmic time, which could explain an accretion history with a succession of 'on and off' phases. In the observations presented here we can only detect very bright objects. Further investigations at larger telescopes (e.g., with the GROND-descendant SCORPIO instrument at Gemini Observatory) will shed new light onto the degenerate scenarios to explain the absence of QSO pairs with $M_{1450}(\mathrm{AB})<-26 \mathrm{mag}$.

\subsection{Incidence of brown dwarfs}

The exposure for the spectroscopic observations was determined to provide $>5 \sigma$ detections of flux redwards of Ly- $\alpha$ (if present) binned at $200 \AA$. Thus, the signal-to-noise ratio of these spectra is good enough to distinguish brown dwarfs and low-redshift galaxies from QSOs, but much too low to perform a spectral classification of the dwarfs or galaxies. We therefore revert to 
J. Greiner et al.: Quasar clustering at redshift 6
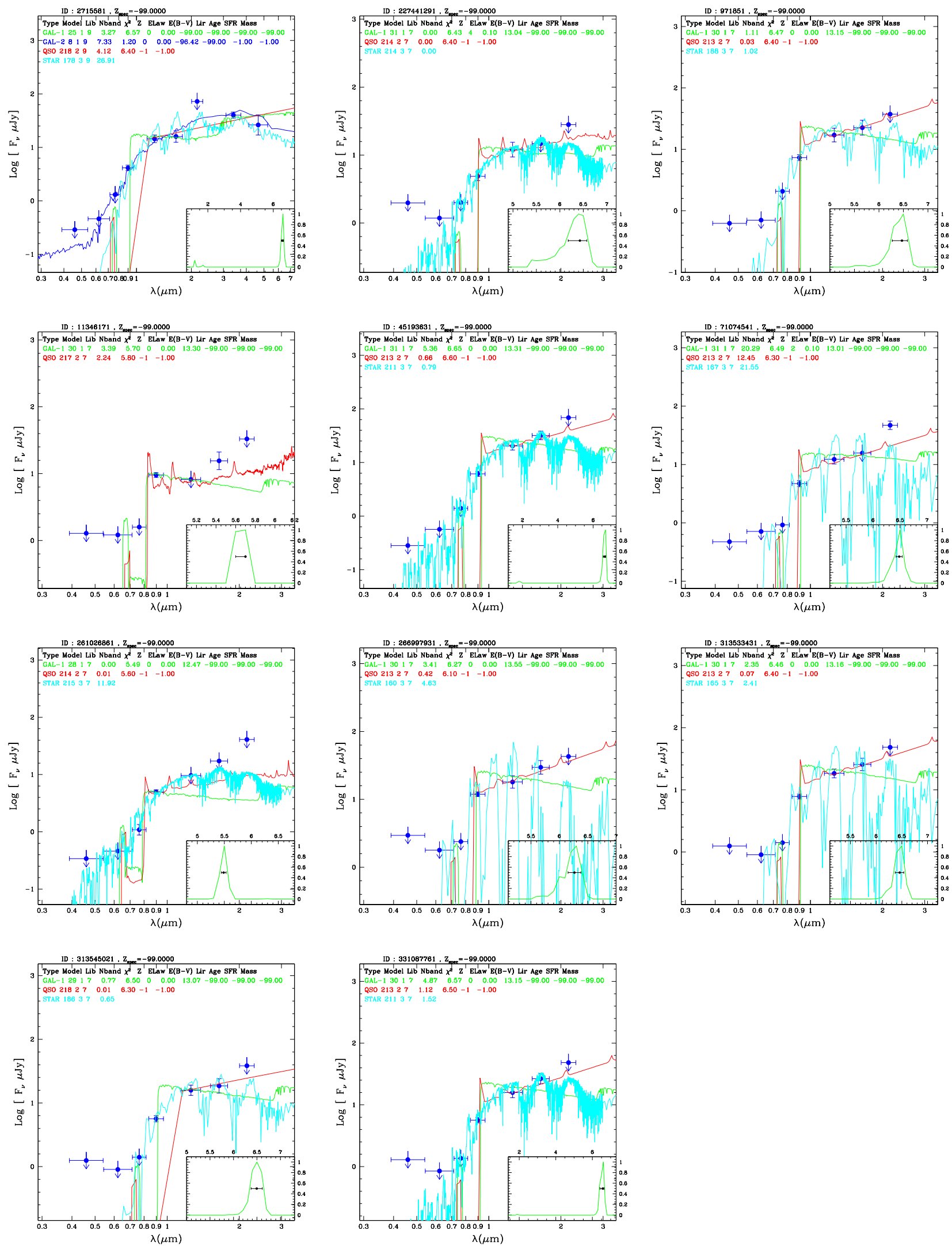

Fig. 6. Le PHARE fits for the ten objects from Tables A.4 and A.5, for which a QSO template provides the better fit, plus the one with a best-fit galaxy template (from top left to bottom right): ULASJ0148+0600, CFHQSJ1509-1749, PSOJ009.7355-10.4316, PSOJ011.3899+09.0325 C2, PSOJ045.1840-22.5408, PSOJ071.0322-04.5591, PSOJ261.0364+19.0286_C1, PSOJ267.0021+22.7812_C2, SDSSJ2054-0005_C3, SDSSJ2054-0005_C4, IMSJ2204+0012. These are the SEDs after correction of the galactic foreground $A_{\mathrm{V}}$, and most of the dwarf fits return an even worse reduced $\chi^{2}$ for the no- $A_{\mathrm{V}}$ SEDs than shown here. 

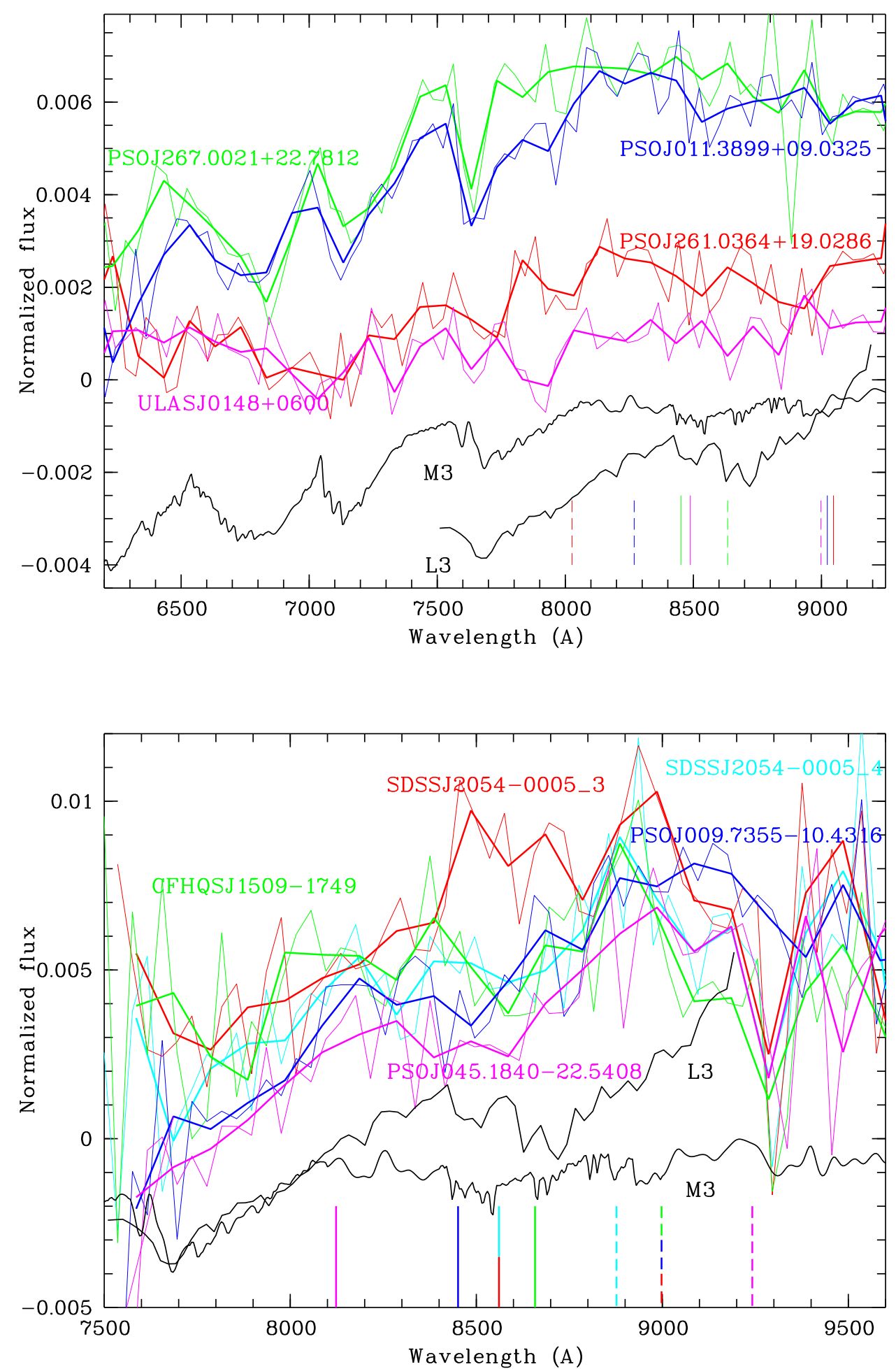

Fig. 7. Long-slit spectra binned at 160 and $400 \AA$ (thin and thick lines, respectively) obtained with the GMOS spectrographs at the Gemini North (top panel) and South (lower panel) Observatories. The vertical solid lines denote the position of Ly- $\alpha$ of its 'mother' QSO, the vertical dashed line the position of Ly- $\alpha$ based on the Le PHARE fits. The dwarf templates are at arbitrary normalizations.

the results of the LePHARE fits, and provide the spectral types of the best-fitting template in the last columns of Tables. A.4 and A.5. For the conversion from the Le PHARE best-fit temperature to spectral type we used the mean value from the compilation of Kellogg (2017). The associated error is \pm 2 subtypes over most of the range.

Since the unWISE (Schlafly et al. 2019) and CatWISE (Marocco et al. 2021) catalogues became available after the selection of our targets for spectroscopic follow-up, we ran a new cross-correlation of our targets against these catalogues.
From our list of 17 candidates without ALLWISE counterparts (Table A.5) we found 6 objects with W1 and W2 entries. Rerunning Le PHARE fits with this extended photometry yields consistent results for three dwarf identifications (within the above-mentioned \pm 2 sub-type error), but deviates for the other three objects: two SEDs, previously best fit with a QSO or M8 dwarf template are now best fit with a low-redshift galaxy $(z \sim$ 1.2 in both cases), similar to ULASJ0148+0600 (Table A.4), and another source, SDSSJ2054-0005_3, for which the best-fit template is a galaxy at redshift 6 , which is inconsistent with our 
absolute photometry. The second-best template (nearly identical $\chi^{2}$ ) is a QSO at redshift 6, which is unlikely given our GMOS spectrum, and the third-best template is an L2 dwarf, but with substantially worse $\chi^{2}$. We therefore do not assign an identification in Table A.6. For the candidate PSOJ055.4244-00.8035, which also had a QSO template as best fit in Le PHARE, but for which we did not obtain a spectrum, we refrain from changing the ID, though Occam's razor would argue against a QSO.

The combination of our spectroscopic results and the Le PHARE fits results in the following identifications: 20 dwarfs, 3 low-redshift galaxies, 1 QSO, and 2 unsettled cases (Tables A.4-A.6). Their spectral range is rather wide, from M5 to T8, though accurate spectral typing remains to be done. The large incidence rate of such dwarfs is a surprise because our search was not tuned towards late dwarfs, and also because our small search size was just 0.52 square degrees (summing over all 116 candidates). For instance, Kakazu et al. (2010) found six faint $(19 \lesssim J \lesssim 20)$ ultracool T dwarfs over a $9.3 \mathrm{deg}^{2}$ area with a limiting magnitude of $z^{\prime}(\mathrm{AB}) \lesssim 23.3 \mathrm{mag}$. This is slightly deeper than our mean depth of $z^{\prime}(\mathrm{AB}) \lesssim 23.1 \mathrm{mag}$, yet we find two $\mathrm{T}$ dwarfs per $0.52 \mathrm{deg}^{2}$, compared to their $0.6 \mathrm{deg}^{-2}$, a factor of 6 higher. Given that we pointed at known high- $z$ QSOs, our search area similarly avoids the galactic plane. In a more recent compilation, Ryan \& Reid (2016) used thick and thin exponential disc models and the luminosity function of ultracool dwarfs to predict their surface density per spectral type. While this depends strongly on the galactic coordinates, the prediction for $\mathrm{T}$ dwarfs has only a small dependence on galactic latitude, suggesting an even higher excess in our data, about a factor of 20, with even the Kakazu et al. (2010) surface density being higher by a factor of $\sim 3$. For early L-type dwarfs, the excess is smaller, and for the M8-9 dwarfs we find consistency. Figure 5 summarises the findings.

Since we observe a substantial excess of $\mathrm{T}$ dwarfs, search completeness cannot be an issue. Foreground extinction correction should also not be an issue since most of these dwarfs are within approximately $100 \mathrm{pc}$ distance. Even if we adopt (systematic?) error in the temperature estimates from the Le PHARE fits that is twice as large, this would not change our numbers. One potential reason could be the appropriateness of the templates in Le PHARE, meaning that dwarfs are more numerous than lowredshift red galaxies, for example. This is contrary to the case of high- $z$ QSOs, for which late dwarfs form a numerous class of false positives. This finding of a higher than expected T-dwarf number density is a surprising puzzle that warrants further study. Existing GROND data could provide a first step in this direction, though spectroscopic typing of the candidate dwarfs is the ultimate step.

\section{Conclusions}

No candidate QSO pair has been found in a search of 116 spectroscopically confirmed redshift 6 quasars within our $0.1-$ $3.3 h^{-1} \mathrm{cMpc}$ search radius with a companion brighter than $M_{1450}(\mathrm{AB})<-26 \mathrm{mag}$. Although this result is consistent with cosmological-scale galaxy evolution simulations (given the redshift, search volume, and luminosity limits), the serendipitously discovered pairs at $z=4.26$ (Schneider et al. 2000) and $z=5.02$ (McGreer et al. 2016) suggested one pair every 40 QSOs, or $2.5 \%$, implying an empirical expectation of 3 pairs in our sample of 116 search targets. At first glance this result suggests little clustering at redshift 6 . However, with updated knowledge of the faint end of the luminosity function, the $w_{\mathrm{p}}$ statistics returns more than an order of magnitude higher value than inferred from the low-redshift clustered QSOs; thus, given the still low statistics in our search and the uncertainties (in particular in the unknown search volumes) of the serendipitous detections, we are not able to claim that the clustering at redshift 6 is indeed smaller than that up to redshift 5 .

As a consequence of our search, we find a higher than expected rate of ultracool dwarfs. While the excess of L dwarfs is marginally consistent with statistics and could be due to the accidental lack of a single source about 1 mag fainter, this argument does not hold for the T dwarfs. The observed excess of a factor of 20 suggests (i) strong surface density variations over the sky, (ii) a lack of appropriate templates for other source types (e.g., low-redshift red galaxies) such that the $\mathrm{T}$ templates just happen to be the closest in shape to the observed SEDs, or (iii) an underestimate of the true rate of T dwarfs. A larger survey at the same depth would be needed to settle this question.

Acknowledgements. We thank the referee for the detailed comments. Part of the funding for GROND (both hardware as well as personnel) was generously granted from the Leibniz-Prize to Prof. G. Hasinger (DFG grant HA 1850/28-1). P. S. acknowledges support through the Sofja Kovalevskaja Award from the Alexander von Humboldt Foundation of Germany while at MPE. J. G. greatly acknowledges the GROND observers Thomas Krühler, Tassilo Schweyer, Simon Steinmassl, Helmut Steinle and Phil Wiseman. We explicitly acknowledge the open attitude of providing the detailed model predictions for dwarfs by Ryan \& Reid (2016) for download, which only enabled the creation of Fig. 5. The reported spectroscopy is based on observations obtained at the international Gemini Observatory, a program of NSF's NOIRLab, which is managed by the Association of Universities for Research in Astronomy (AURA) under a cooperative agreement with the National Science Foundation on behalf of the Gemini Observatory partnership: the National Science Foundation (United States), National Research Council (Canada), Agencia Nacional de Investigación y Desarrollo (Chile), Ministerio de Ciencia, Tecnología e Innovación (Argentina), Ministério da Ciência, Tecnologia, Inovações e Comunicações (Brazil), and Korea Astronomy and Space Science Institute (Republic of Korea). This paper includes data gathered with the $6.5 \mathrm{~m}$ Magellan Telescopes located at Las Campanas Observatory, Chile. This research has made use of the NASA/IPAC Infrared Science Archive, which is operated by the Jet Propulsion Laboratory, California Institute of Technology, under contract with the National Aeronautics and Space Administration. For photometric calibration, the Sloan Digital Sky Survey, PanSTARRS, SkyMapper and 2MASS are acknowledged: Funding for SDSS-III has been provided by the Alfred P. Sloan Foundation, the Participating Institutions, the National Science Foundation, and the US Department of Energy Office of Science. The SDSS-III web site is http://www . sdss3. org/. SDSS-III is managed by the Astrophysical Research Consortium for the Participating Institutions of the SDSS-III Collaboration including the University of Arizona, the Brazilian Participation Group, Brookhaven National Laboratory, Carnegie Mellon University, University of Florida, the French Participation Group, the German Participation Group, Harvard University, the Instituto de Astrofisica de Canarias, the Michigan State/Notre Dame/JINA Participation Group, Johns Hopkins University, Lawrence Berkeley National Laboratory, Max Planck Institute for Astrophysics, Max Planck Institute for Extraterrestrial Physics, New Mexico State University, New York University, Ohio State University, Pennsylvania State University, University of Portsmouth, Princeton University, the Spanish Participation Group, University of Tokyo, University of Utah, Vanderbilt University, University of Virginia, University of Washington, and Yale University. The Pan-STARRS1 Surveys (PS1) have been made possible through contributions of the Institute for Astronomy, the University of Hawaii, the Pan-STARRS Project Office, the Max-Planck Society and its participating institutes, the Max Planck Institute for Astronomy, Heidelberg and the Max Planck Institute for Extraterrestrial Physics, Garching, The Johns Hopkins University, Durham University, the University of Edinburgh, Queen's University Belfast, the Harvard-Smithsonian Center for Astrophysics, the Las Cumbres Observatory Global Telescope Network Incorporated, the National Central University of Taiwan, the Space Telescope Science Institute, the National Aeronautics and Space Administration under Grant No. NNX08AR22G issued through the Planetary Science Division of the NASA Science Mission Directorate, the National Science Foundation under Grant No. AST-1238877, the University of Maryland, and Eotvos Lorand University (ELTE). The national facility capability for SkyMapper has been funded through ARC LIEF grant LE130100104 from the Australian Research Council, awarded to the University of Sydney, the Australian National University, Swinburne University of Technology, the University of Queensland, the University of Western Australia, the University of Melbourne, Curtin University of Technology, Monash University 
and the Australian Astronomical Observatory. SkyMapper is owned and operated by The Australian National University's Research School of Astronomy and Astrophysics. The survey data were processed and provided by the SkyMapper Team at ANU. The SkyMapper node of the All-Sky Virtual Observatory (ASVO) is hosted at the National Computational Infrastructure (NCI). Development and support the SkyMapper node of the ASVO has been funded in part by Astronomy Australia Limited (AAL) and the Australian Government through the Commonwealth's Education Investment Fund (EIF) and National Collaborative Research Infrastructure Strategy (NCRIS), particularly the National eResearch Collaboration Tools and Resources (NeCTAR) and the Australian National Data Service Projects (ANDS). This publication makes use of data products from the Two Micron All Sky Survey, which is a joint project of the University of Massachusetts and the Infrared Processing and Analysis Center/California Institute of Technology, funded by the National Aeronautics and Space Administration and the National Science Foundation.

\section{References}

Arnouts, S., Cristiani, S., Moscardini, L., et al. 1999, MNRAS, 310, 540 Ayromlou, M., Nelson, D., Yates, R. M., et al. 2021a, MNRAS, 502, 1051 Ayromlou, M., Kauffmann, G., Yates, R. M., et al. 2021b, MNRAS, 505, 492 Bañados, E., Venemans, B. P., Decarli, R., et al. 2016, ApJS, 227, 11 Bañados, E., Venemans, B. P., Mazzucchelli, C., et al. 2018, Nature, 553, 473 Begelman, M. C., Volonteri, M., \& Rees, M. J. 2006, MNRAS, 370, 289 Bhowmick, A. K., DiMatteo, T., Eftekharzadeh, S., \& Myers, A. D. 2019, MNRAS, 485, 2026

Bonoli, S., Marulli, F., Springel, V., et al. 2009, MNRAS, 396, 423

Bromm, V., \& Loeb, A. 2003, ApJ, 596, 34

Carnall, A. C., Shanks, T., Chehade, B., et al. 2015, MNRAS, 451, L16

Chambers, K. C., Magnier, E. A., Metcalfe, N., et al. 2016, ArXiv e-prints [arXiv: 1612.05560 ]

Chehade, B., Shanks, T., Findlay, J., et al. 2016, MNRAS, 459, 1179

Connor, T., Bañados, E., Mazzucchelli, C., et al. 2020, ApJ, 900, 189

Costa, T., Sijacki, D., Trenti, M., \& Haehnelt, M. G. 2014, MNRAS, 439, 2146

Croton, D. J., Springel, V., White, S. D. M., et al. 2006, MNRAS, 365, 11

Davis, M., \& Peebles, P. J. E. 1983, ApJ, 267, 465

Decarli, R., Walter, F., Venemans, B. P., et al. 2017, Nat, 545, 457

DeGraf, C., \& Sijacki, D. 2017, MNRAS, 466, 3331

Devecchi, B., \& Volonteri, M. 2009, ApJ, 694, 302

Dijkstra, M., Haiman, Z., Mesinger, A., \& Wyithe, J. S. B. 2008, MNRAS, 391, 1961

Di Matteo, T., Croft, R. A. C., Feng, Y., et al. 2017, MNRAS, 467, 4243

Djorgovski, S. G., Stern, D., Mahabal, A. A., \& Brunner, R. 2003, ApJ, 596, 67 Duras, F., Bongiorno, A., Ricci, F., et al. 2020, A\&A, 636, 73

Eftekharzadeh, S., Myers, A. D., Hennawi, J. F., et al. 2017, MNRAS, 468, 77

Eisenstein, D. J., Weinberg, D. H., Agol, E., et al. 2011, AJ, 142, 72

Fan, X., Strauss, M. A., Schneider, D. P., et al. 1999, AJ, 118, 1

Fang, L. Z. 1989, Int. J. Mod. Phys. A, 4, 3477

Greiner, J., Bornemann, W., Clemens, C., et al. 2008, PASP, 120, 405

Habouzit, M., Volonteri, M., Latif, M., Dubois, Y., \& Peirani, S. 2016a, MNRAS 463,529

Habouzit, M., Volonteri, M., Latif, M., et al. 2016b, MNRAS, 456, 1901

Habouzit, M., Volonteri, M., Somerville, R. S., et al. 2019, MNRAS, 489, 1206

Habouzit, M., Li, Y., Somerville, R. S., et al. 2021, MNRAS, 503, 1940

Haiman, Z., \& Hui, L. 2001, ApJ, 547, 27

Haehnelt, M., \& Nusser, A. , 1999, in Evolution of large scale structure: from recombination to Garching, Proc. MPA-ESO cosmology conf., August 1998, eds. A. J.Banday et al., 375

Hennawi, J. F., Strauss, M. A., Oguri, M., et al. 2006, AJ, 131, 1

Hennawi, J. F., Myers, A. D., Shen, Y., et al. 2010, ApJ, 719, 1672

Henriques, B. M. B., Yates, R. M., Fu, J., et al. 2020, MNRAS, 491, 5795

Hopkins, P. F., Lidz, A., Hernquist, L., et al. 2007, ApJ, 662, 110

Husband, K., Bremer, M. N., Stanway, E. R., et al. 2013, MNRAS, 432, 2869

Ilbert, O., Arnouts, S., McCracken, H. J., et al. 2006, A\&A, 457, 841

Inayoshi, K., Visbal, E., \& Haiman, Z. 2020, ARAA, 58, 27

Johnson, J. L., Whalen, D. J., Li, H., \& Holz, D. E. 2013, ApJ, 771, 116

Jiang, L., Fan, X., Bian, F., et al. 2009, AJ, 138, 305

Jiang, L., McGreer, I. D., Fan, X., et al. 2016, ApJ, 833, 222

Kakazu, Y., Hu, E. M., Liu, M. C., et al. 2010, ApJ, 723, 184

Kashikawa, N., Kitayama, T., Doi, M., et al. 2007, ApJ, 663, 765

Kellogg, K. 2017, Electronic Thesis and Dissertation Repository, 4857, https: //ir.lib.uwo.ca/etd/4857
Kim, S., Stiavelli, M., Trenti, M., et al. 2009, ApJ, 695, 809

Kim, Y., Im, M., Jeon, Y., et al. 2015, ApJ, 813, L35

Kroupa, P., Subr, L., Jerabkova, T., \& Wang, L. 2020, MNRAS, 498, 5652

Krühler, T., Küpcü, Yoldaş A., Greiner, J., et al. 2008, ApJ, 685, 376

Kulkarni, G., Worseck, G., \& Hennawi, J. F. 2019, MNRAS, 488, 1035

Küpcü, Yoldaş A., Krühler, T., Greiner, J., et al. 2008, AIP Conf. Proc., 1000, 227

Latif, M. A., \& Volonteri, M. 2015, MNRAS, 452, 1026

Leśniewska, A., \& Michalowski, M. J. 2019, A\&A, 624, L13

Lodato, G., \& Narayan, P. 2006, MNRAS, 371, 1813

Loeb, A., \& Rasio, F. A. 1994, ApJ, 432, 52

Lupi, A., Volonteri, M., Decarli, R., et al. 2019, MNRAS, 488, 4004

Madau, P., \& Rees, M. J. 2001, ApJ, 551, L27

Madau, P., Haardt, F., \& Rees, M. J. 1999, ApJ, 514, 648

Mainzer, A., Bauer, J., Grav, T., et al. 2011, ApJ, 731, 53

Marshall, M. A., Ni, Y., Di Matteo, T., et al. 2020, MNRAS, 499, 3891

Marinacci, F., Vogelsberger, M., Pakmor, R., et al. 2018, MNRAS, 480, 5113

Marocco, F., Eisenhardt, P. R. M., Fowler, J. W., et al. 2021, ApJS, 253, 8

Matsuoka, Y., Onoue, M., Kashikawa, N., et al. 2016, ApJ, 828, 26

Matsuoka, Y., Strauss, M. A., Kashikawa, N., et al. 2018, ApJ, 869, 150

Mazzucchelli, C., Bañados, E., Venemans, B. P., et al. 2017a, ApJ, 849, 91

Mazzucchelli, C., Bañados, E., Decarli, R., et al. 2017b, ApJ, 834, 83

McGreer, I. D., Eftekharzadeh, S., Myers, A. D., \& Fan, X. 2016, AJ, 151, 61

Naiman, J. P., Pillepich, A., Springel, V., et al. 2018, MNRAS, 477, 1206

Neeleman, M., Bañados, E., Walter, F., et al. 2019, ApJ, 882, 10

Nelson, D., Pillepich, A., Springel, V., et al. 2018, MNRAS, 475, 624

Omukai, K., Schneider, R., \& Haiman, Z. 2008, ApJ, 686, 801

Pillepich, A., Springel, V., Nelson, D., et al. 2018, MNRAS, 473, 4077

Planck Collaboration XIII. 2016, A\&A, 594, A13

Porciani, C., Magliocchetti, M., \& Norberg, P. 2007, MNRAS, 355, 1010

Reed, S. L., McMahon, R. G., Banerji, M., et al. 2015, MNRAS, 454, 3952

Regan, J. A., \& Haehnelt, M. G. 2009, MNRAS, 393, 858

Ross, N. P., McGreer, I. D., White, M., et al. 2013, ApJ, 773, 14

Ryan, R. E., \& Reid, I. N. 2016, AJ, 151, 92

Schlafly, E. F., \& Finkbeiner, D. P. 2011, ApJ, 737, 103

Schlafly, E. F., Meisner, A. M., \& Green, G. M. 2019, ApJS, 240, 30

Schneider, D. P., Fan, X., Strauss, M. A., et al. 2000, AJ, 120, 2183

Scolnic, D., Casertano, S., Riess, A., et al. 2015, ApJ, 815, 117

Shen, Y., Strauss, M. A., Oguri, M., et al. 2007, AJ, 133, 2222

Shen, Y., Hennawi, J. F., Shankar, F., et al. 2010, ApJ, 719, 1693

Shen, X., Hopkins, P. F., Faucher-Giguee, C. A., et al. 2020, MNRAS, 495, 3252

Sheth, R. K., Mo, H. J., \& Tormen, G. 2001, MNRAS, 323, 1

Silverman, J. D., Tang, S., Lee, K.-G., et al. 2020, ApJ, 899, 154

Skrutskie, M. F., Cutri, R. M., Stiening, R., et al. 2006, AJ, 131, 1163

Songaila, A. 2004, AJ, 127, 2598

Spaans, M., \& Silk, J. 2006, ApJ, 652, 902

Springel, V., White, S. D. M., Jenkins, A., et al. 2005, Nature, 435, 629

Springel, V., Pakmor, R., Pillepich, A., et al. 2018, MNRAS, 475, 676

Tenneti, A., Wilkins, S. M., Di Matteo, T., et al. 2019, MNRAS, 483, 1388

Tody, D. 2019, ASP Conf. Ser., 52, 173

Utsumi, Y., Goto, T., Kashikawa, N., et al. 2010, ApJ, 721, 1680

Valiante, R., Agrarval, B., Habouzit, M., \& Pezzulli, E. 2017, PASA, 34

Valiante, R., Schneider, R., Graziani, L., \& Zappacosta, L. 2018, MNRAS, 474 3825

Venemans, B. P., Findlay, J. R., Sutherland, W. J., et al. 2013, ApJ, 779, 24

Venemans, B. P., Verdoes Kleijn, G. A., Mwebaze, J., et al. 2015, MNRAS, 453, 2259

Visbal, E., Haiman, Z., \& Bryan, G. L. 2014, MNRAS, 445, 1056

Vito, F., Brandt, W. N., Ricci, F., et al. 2021, A\&A, 649, A133

Volonteri, M. 2010, A\&ARv, 18, 279

Volonteri, M., Haardt, F., \& Madau, P. 2003a, ApJ, 582, 559

Volonteri, M., Madau, P., \& Haardt, F. 2003b, ApJ, 594, 661

Volonteri, M., Dubois, Y., Pichon, C., \& Devriendt, J. 2016, MNRAS, 460, 2979

Wang, F., Yang, J., Fan, X., et al. 2021, ApJ, 907, L1

Willott, C. J., Delorme, P., Omont, A., et al. 2007a, AJ, 134, 2435

Willott, C. J., Delorme, P., Reyle, C., et al. 2007b, AJ, 137, 3541

Willott, C. J., Albert, L., Arzoumanian, D., et al. 2010, AJ, 140, 546

Wolf, C., Onken, C. A., Luvaul, L. C., et al. 2018, PASA, 35, 10

Wright, E. L., Eisenhardt, P. R. M., Mainzer, A. K., et al. 2010, AJ, 140, 1868

Wyithe, J. S. B., Loeb, A., \& Carilli, C. 2005, ApJ, 628, 575

Yang, J., Wang, F., Fan, X., et al. 2020, ApJ, 897, L14

Yue, B., Ferrara, A., Salvaterra, R., et al. 2014, MNRAS, 440, 1263

Zeimann, G. R., White, R. L., Becker, R. H., et al. 2011, ApJ, 736, 57 


\section{Appendix A: Additional tables}

Table A.1. Details of the GROND observations of selected known QSOs, sorted by right ascension².

\begin{tabular}{|c|c|c|c|c|c|c|}
\hline Name & $\mathrm{z}$ & $\begin{array}{c}\mathrm{z}_{c a t}^{\prime}(\mathrm{AB}) \\
(\mathrm{mag})\end{array}$ & Obs. date & OB & limiting $\mathrm{z}^{\prime}$ calib & $\begin{array}{c}\mathrm{A}_{\mathrm{V}} \\
(\mathrm{mag})\end{array}$ \\
\hline PSOJ000.3401+26.8358 & 5.75 & $19.28 \pm 0.02$ & $2016-10-25$ & $2 \_1$ & $22.6 \mathrm{~S}$ & 0.11 \\
\hline SDSSJ0005-0006 & 5.85 & $20.44 \pm 0.04$ & 2016-08-29 & 1_1 & $23.3 \mathrm{~S}$ & 0.09 \\
\hline PSOJ002.1073-06.4345 & 5.93 & $20.25 \pm 0.03$ & 2013-09-09 & 1_123 & $22.8 \mathrm{~S}$ & 0.09 \\
\hline PSOJ004.3936+17.0862 & 5.80 & $20.82 \pm 0.07$ & $2016-10-25$ & $\overline{1} \_1$ & $22.6 \mathrm{~S}$ & 0.17 \\
\hline PSOJ004.8140-24.2991 & 5.68 & $19.62 \pm 0.02$ & 2013-09-09 & $1 \_12$ & $22.4 \mathrm{P}$ & 0.04 \\
\hline PSOJ007.0273+04.9571 & 6.00 & $20.56 \pm 0.05$ & 2013-01-14 & 2_12 & $22.9 \mathrm{~S}$ & 0.04 \\
\hline CFHQSJ0033-0125 ${ }^{a}$ & 6.13 & $22.44 \pm 0.08$ & 2016-08-26 & $1 \_1$ & $23.0 \mathrm{~S}$ & 0.06 \\
\hline PSOJ009.3573-08.1190 & 5.72 & $19.91 \pm 0.03$ & 2015-11-05 & 1_1 & $23.2 \mathrm{~S}$ & 0.11 \\
\hline PSOJ009.7355-10.4316 & 5.95 & $20.82 \pm 0.04$ & 2014-12-14 & 2_12 & $23.1 \mathrm{~S}$ & 0.08 \\
\hline PSOJ011.3899+09.0325 & 6.42 & $>22.33$ & $2016-09-20$ & $1 \_12$ & $23.1 \mathrm{~S}$ & 0.16 \\
\hline CFHQSJ0055+0146 ${ }^{k}$ & 6.01 & $22.19 \pm 0.06$ & 2016-09-01 & $1 \_1$ & $24.1 S$ & 0.06 \\
\hline SDSSJ0100+2802 & 6.30 & $18.61 \pm 0.01$ & 2016-08-31 & $1 \_1$ & $23.3 \mathrm{~S}$ & 0.15 \\
\hline CFHQSJ0102-0218 & 5.95 & $22.30 \pm 0.08$ & 2016-08-18 & $1 \_1$ & $23.0 \mathrm{~S}$ & 0.11 \\
\hline PSOJ021.4213-25.8822 & 5.79 & $19.66 \pm 0.03$ & 2014-09-21 & $1 \_1$ & $22.7 \mathrm{P}$ & 0.04 \\
\hline SDSSJ0129-0035 & 5.78 & $22.16 \pm 0.11$ & 2016-09-02 & 1_3 & $23.4 \mathrm{~S}$ & 0.09 \\
\hline PSOJ023.0071-02.2675 & 5.90 & $20.19 \pm 0.03$ & 2013-09-10 & $2 \_12$ & $23.4 \mathrm{~S}$ & 0.09 \\
\hline CFHQSJ0136+0226 & 6.21 & $22.06 \pm 0.16$ & 2016-09-02 & 1_1 & & 0.11 \\
\hline PSOJ025.2376-11.6831 & 5.85 & $20.18 \pm 0.02$ & 2013-09-08 & 1_123 & $22.9 \mathrm{P}$ & 0.06 \\
\hline ATLASJ025.6821-33.4627 ${ }^{l}$ & 6.31 & $19.63 \pm 0.08$ & 2016-08-18 & $\overline{1} \_1$ & 22.3SM & 0.07 \\
\hline ULASJ0148+0600 & 5.98 & $19.45 \pm 0.01$ & 2016-08-31 & 1 & $23.7 \mathrm{~S}$ & 0.16 \\
\hline PSOJ029.5172-29.0886 & 5.99 & $19.48 \pm 0.02$ & 2013-09-10 & $1 \_12$ & $22.8 \mathrm{P}$ & 0.04 \\
\hline ATLASJ029.9915-36.5658 ${ }^{l}$ & 6.02 & $19.54 \pm 0.08$ & 2016-08-18 & 1_1 & $22.4 \mathrm{SM}$ & 0.04 \\
\hline ULASJ0203+0012 & 5.72 & $20.74 \pm 0.06$ & $2016-08-29$ & $1 \_1$ & $23.1 \mathrm{~S}$ & 0.07 \\
\hline CFHQSJ0210-0456 ${ }^{m}$ & 6.43 & $22.67 \pm 0.05$ & 2016-09-02 & $1 \_1$ & $23.4 \mathrm{~S}$ & 0.05 \\
\hline CFHQSJ0221-0802 ${ }^{m}$ & 6.16 & $22.63 \pm 0.05$ & 2016-08-18 & $1 \_1$ & 23.0S & 0.08 \\
\hline PSOJ036.5078+03.0498 & 6.54 & $21.44 \pm 0.12$ & 2016-08-29 & $1 \_1$ & $23.1 \mathrm{~S}$ & 0.09 \\
\hline CFHQSJ0227-0605 ${ }^{k}$ & 6.20 & $21.71 \pm 0.06$ & 2016-09-01 & 1_1 & $23.6 \mathrm{~S}$ & 0.09 \\
\hline PSOJ037.9706-28.8389 & 6.00 & $20.73 \pm 0.06$ & 2013-01-17 & $1 \_123$ & $23.4 \mathrm{P}$ & 0.04 \\
\hline SDSSJ0239-0045 & 5.82 & $22.08 \pm 0.11$ & 2016-08-18 & 1_1 & $22.8 \mathrm{~S}$ & 0.08 \\
\hline PSOJ040.0159+17.5458 & 5.68 & $20.60 \pm 0.05$ & $2016-08-30$ & $2 \_1$ & $23.8 \mathrm{P}$ & 0.23 \\
\hline PSOJ042.6690-02.9174 & 5.89 & $20.49 \pm 0.05$ & 2013-09-10 & $1 \_\overline{1} 23$ & $23.4 \mathrm{~S}$ & 0.12 \\
\hline PSOJ045.1840-22.5408 & 5.68 & $20.34 \pm 0.05$ & 2013-01-14 & $1 \_123$ & $23.2 \mathrm{P}$ & 0.07 \\
\hline SDSSJ0303-0019 & 6.08 & $20.99 \pm 0.06$ & 2016-08-30 & 1_1 & $23.9 \mathrm{~S}$ & 0.29 \\
\hline VIKJ0305-3150 ${ }^{b}$ & 6.60 & $22.12 \pm 0.07$ & 2016-09-02 & $12 \_1$ & 23.7SM & 0.03 \\
\hline CFHQSJ0316-1340 & 5.99 & $21.57 \pm 0.12$ & $2016-08-30$ & $1]$ & $24.1 \mathrm{~S}$ & 0.14 \\
\hline PSOJ049.2934-26.5543 & 5.94 & $20.77 \pm 0.06$ & $2016-08-31$ & $2 \_1$ & $23.4 \mathrm{P}$ & 0.05 \\
\hline VIKJ0328-3253 ${ }^{i}$ & 5.86 & $19.83 \pm 0.02$ & 2016-08-21 & $1 \_1$ & $23.1 \mathrm{P}$ & 0.03 \\
\hline PSOJ053.9605-15.7956 & 5.87 & $20.34 \pm 0.04$ & $2016-08-21$ & $1 \_1$ & $23.3 \mathrm{P}$ & 0.24 \\
\hline PSOJ055.4244-00.8035 & 5.68 & $20.19 \pm 0.04$ & 2014-02-02 & $1 \_1$ & $22.6 \mathrm{~S}$ & 0.25 \\
\hline PSOJ056.7168-16.4769 & 5.99 & $20.00 \pm 0.04$ & 2016-08-19 & $1 \_1$ & $22.8 \mathrm{P}$ & 0.14 \\
\hline SDSSJ0353+0104 & 6.07 & $20.81 \pm 0.07$ & $2016-08-15$ & 1 & $23.4 \mathrm{~S}$ & 0.78 \\
\hline PSOJ060.5529+24.8567 & 6.18 & $20.18 \pm 0.03$ & 2014-12-16 & $1 \_12$ & $23.0 \mathrm{P}$ & 0.64 \\
\hline PSOJ065.4085-26.9543 & 6.14 & $20.48 \pm 0.05$ & 2014-02-05 & 2 & $22.8 \mathrm{P}$ & 0.11 \\
\hline PSOJ065.5041-19.4579 & 6.12 & $19.79 \pm 0.03$ & 2014-12-17 & $1 \_1$ & $22.8 \mathrm{P}$ & 0.10 \\
\hline
\end{tabular}

Notes. ${ }^{2}$ The redshift and the observed $z^{\prime}$ magnitude are taken from the original discovery papers, predominantly Bañados et al. (2016) unless marked as follows: ${ }^{a}=$ Willott et al. (2007a), ${ }^{b}=$ Venemans et al. (2013), ${ }^{c}=$ Jiang et al. (2016), ${ }^{d}=$ Mazzucchelli et al. $(2017 \mathrm{~b}),{ }^{e}=$ Zeimann et al. (2011), ${ }^{f}=$ Matsuoka et al. (2016), ${ }^{g}=$ Kim et al. (2015), ${ }^{h}=$ Reed et al. (2015), ${ }^{i}=$ Venemans et al. (2015), ${ }^{j}=$ Jiang et al. $(2009),{ }^{k}=$ Willott et al. (2007b), ${ }^{l}=$ Carnall et al. (2015), ${ }^{m}=$ Willott et al. (2010). Column 4 is the date at the start of the observing night, and Col. 5 indicates the nth observing night of that target (first number) and which observation blocks (OBs) were used in the analysis (numbers after the underscore): more than one number indicates stacking of multiple OBs. Column 6 provides the $3 \sigma$ limiting $z^{\prime}$ magnitude of the (stacked) observation, not corrected for galactic foreground extinction (which is given in Col. 7, based on Schlafly \& Finkbeiner 2011). The letter attached to the $z^{\prime}$ limiting magnitude denotes the catalogue used for photometric calibration of the optical channels $g^{\prime} r^{\prime} i^{\prime} z^{\prime}: S=S D S S$ (DR12), P=PanSTARRS1, SM=SkyMapper (DR1.1). 
Table A.1. continued.

\begin{tabular}{|c|c|c|c|c|c|c|}
\hline Name & $\mathrm{z}$ & $\begin{array}{c}\mathrm{z}_{c a t}^{\prime}(\mathrm{AB}) \\
(\mathrm{mag})\end{array}$ & Obs. date & OB & limiting $\mathrm{z}^{\prime}$ calib & $\begin{array}{c}\mathrm{A}_{\mathrm{V}} \\
(\mathrm{mag}) \\
\end{array}$ \\
\hline PSOJ071.0322-04.5591 & 5.89 & $20.30 \pm 0.04$ & 2015-11-06 & $1 \_12$ & $23.6 \mathrm{~S}$ & 0.12 \\
\hline PSOJ071.4507-02.3332 & 5.69 & $19.18 \pm 0.01$ & $2016-08-20+30$ & 12_1 & $23.6 \mathrm{P}$ & 0.10 \\
\hline DESJ0454-4448 ${ }^{h}$ & 6.10 & $20.20 \pm 0.01$ & 2016-08-21 & 1 & $23.4 \mathrm{SM}$ & 0.03 \\
\hline PSOJ075.9356-07.5061 & 5.88 & $20.33 \pm 0.05$ & 2017-09-18 & $2-1$ & $23.6 \mathrm{P}$ & 0.52 \\
\hline PSOJ089.9394-15.5833 & 6.05 & $19.66 \pm 0.03$ & 2015-11-08 & 1_12 & $23.4 \mathrm{P}$ & 0.79 \\
\hline PSOJ108.4429+08.9257 & 5.92 & $19.45 \pm 0.02$ & 2015-11-08 & $1 \_1$ & $22.8 \mathrm{P}$ & 0.23 \\
\hline SDSSJ0818+1722 & 6.02 & $19.55 \pm 0.02$ & $2017-01-26$ & 1 & $22.9 \mathrm{~S}$ & 0.09 \\
\hline ULASJ0828+2633 & 6.05 & $20.72 \pm 0.06$ & 2017-02-07 & $1 \_1$ & $22.5 \mathrm{~S}$ & 0.21 \\
\hline PSOJ127.2817+03.0657 & 5.85 & $20.69 \pm 0.05$ & $2014-12-15$ & $1 \_12$ & $22.8 \mathrm{~S}$ & 0.04 \\
\hline SDSSJ0836+0054 & 5.81 & $18.70 \pm 0.01$ & $2012-01-05$ & 1_12 & $23.3 \mathrm{~S}$ & 0.13 \\
\hline VIKJ0839+0015 ${ }^{i}$ & 5.84 & $21.09 \pm 0.05$ & $2017-01-26$ & 1 & $23.3 \mathrm{~S}$ & 0.12 \\
\hline SDSSJ0842+1218 & 6.07 & $19.83 \pm 0.03$ & $2017-05-02$ & $1 \_2$ & $21.9 \mathrm{~S}$ & 0.18 \\
\hline HSCJ0859+0022 ${ }^{f}$ & 6.39 & $22.77 \pm 0.01$ & $2017-01-25$ & $1 \_1$ & $23.4 \mathrm{~S}$ & 0.08 \\
\hline PSOJ135.3860+16.2518 & 5.63 & $20.67 \pm 0.04$ & 2014-02-04 & 1_1 & $22.8 \mathrm{~S}$ & 0.11 \\
\hline PSOJ135.8704-13.8336 & 5.91 & $20.31 \pm 0.04$ & 2014-12-17 & $12 \_12$ & $23.2 \mathrm{P}$ & 0.18 \\
\hline PSOJ157.9070-02.6599 & 5.88 & $20.24 \pm 0.03$ & $2017-01-25$ & $2 \_1$ & $23.1 \mathrm{~S}$ & 0.12 \\
\hline PSOJ159.2257-02.5438 & 6.38 & $20.46 \pm 0.04$ & $2017-01-26$ & 1 & $23.5 \mathrm{~S}$ & 0.13 \\
\hline SDSSJ1044-0125 & 5.78 & $19.31 \pm 0.01$ & $2017-01-26$ & $1 \_1$ & $24.1 \mathrm{~S}$ & 0.14 \\
\hline CFHQSJ1059-0906 & 5.92 & $20.84 \pm 0.06$ & 2017-01-24 & $1 \_1$ & $23.6 \mathrm{P}$ & 0.09 \\
\hline PSOJ167.6415-13.4960 ${ }^{d}$ & 6.51 & $>22.94$ & 2017-05-02 & $1 \_1$ & $23.4 \mathrm{~S}$ & 0.15 \\
\hline PSOJ174.7920-12.2845 & 5.81 & $20.04 \pm 0.04$ & $2017-01-28$ & $1 \_1$ & $23.4 \mathrm{P}$ & 0.09 \\
\hline PSOJ175.4091-20.2654 & 5.69 & $20.17 \pm 0.04$ & 2017-01-24 & $1 \_1$ & $23.6 \mathrm{P}$ & 0.14 \\
\hline VIKJ1152+0055 & 6.37 & $21.83 \pm 0.01$ & 2014-02-05 & 1_- 123 & $23.3 \mathrm{~S}$ & 0.06 \\
\hline PSOJ183.2991-12.7676 & 5.86 & $19.47 \pm 0.02$ & 2017-04-29 & $1 \_3$ & $23.3 \mathrm{P}$ & 0.13 \\
\hline PSOJ184.3389+01.5284 & 6.20 & $21.20 \pm 0.07$ & $2017-05-01$ & 1_1 & $23.2 \mathrm{P}$ & 0.06 \\
\hline PSOJ187.1047-02.5609 & 5.77 & $20.92 \pm 0.05$ & 2014-01-29 & $1 \_123$ & $23.1 \mathrm{~S}$ & 0.09 \\
\hline PSOJ187.3050+04.3243 & 5.89 & $20.92 \pm 0.04$ & $2017-04-30$ & 1 & $23.4 \mathrm{~S}$ & 0.05 \\
\hline SDSSJ1306+0356 & 6.02 & $19.76 \pm 0.03$ & $2017-04-30$ & $1 \_1$ & $23.2 \mathrm{~S}$ & 0.07 \\
\hline ULASJ1319+0950 & 6.13 & $20.13 \pm 0.02$ & 2017-04-29 & $1 \_1$ & $23.6 \mathrm{~S}$ & 0.05 \\
\hline PSOJ209.2058-26.7083 & 5.72 & $19.35 \pm 0.01$ & 2014-02-02 & 1_12 & $23.2 \mathrm{P}$ & 0.17 \\
\hline PSOJ $210.8297+09.0474$ & 5.88 & $20.31 \pm 0.03$ & $2016-08-23$ & 1 & $22.4 \mathrm{~S}$ & 0.07 \\
\hline PSOJ210.8722-12.0094 & 5.84 & $21.09 \pm 0.07$ & $2018-03-23$ & $23 \_1$ & $23.3 \mathrm{~S}$ & 0.19 \\
\hline PSOJ212.2974-15.9865 & 5.83 & $20.98 \pm 0.06$ & $2015-05-21$ & $1 \_123$ & $23.2 \mathrm{~S}$ & 0.26 \\
\hline SDSSJ1411+1217 & 5.90 & $20.58 \pm 0.02$ & $2016-08-22$ & $\overline{1} \_1$ & $22.2 \mathrm{~S}$ & 0.06 \\
\hline PSOJ213.3629-22.5617 & 5.92 & $19.55 \pm 0.02$ & 2016-08-22 & $2 \_1$ & $22.9 \mathrm{P}$ & 0.23 \\
\hline PSOJ $213.7329-13.4803$ & 5.78 & $20.86 \pm 0.05$ & $2016-08-23$ & 2 & $23.0 \mathrm{P}$ & 0.21 \\
\hline PSOJ215.1514-16.0417 & 5.73 & $19.08 \pm 0.02$ & $2011-03-05$ & 9_- 123 & $22.8 \mathrm{P}$ & 0.21 \\
\hline PSOJ217.0891-16.0453 & 6.11 & $20.46 \pm 0.04$ & $2016-08-22$ & $\overline{2} \_1$ & $22.8 \mathrm{P}$ & 0.25 \\
\hline PSOJ217.9185-07.4120 & 6.14 & $21.10 \pm 0.08$ & 2015-05-19 & 1_- 123 & $23.1 \mathrm{P}$ & 0.18 \\
\hline CFHQSJ1509-1749 ${ }^{a}$ & 6.12 & $20.26 \pm 0.02$ & 2016-08-17 & $\overline{1} \_1$ & $23.2 \mathrm{P}$ & 0.24 \\
\hline PSOJ228.6871+21.2388 & 5.92 & $20.92 \pm 0.06$ & 2017-04-29 & $2 \_1$ & $23.4 \mathrm{~S}$ & 0.13 \\
\hline PSOJ $235.9450+17.0079$ & 5.82 & $20.23 \pm 0.03$ & $2016-08-21$ & $2 \_1$ & $23.1 \mathrm{~S}$ & 0.09 \\
\hline PSOJ236.2912+16.6088 & 5.82 & $20.69 \pm 0.05$ & $2016-08-20$ & $1 \_1$ & $23.0 \mathrm{~S}$ & 0.09 \\
\hline PSOJ238.8510-06.8976 & 5.81 & $20.43 \pm 0.05$ & $2015-05-21$ & $1 \_12$ & $22.7 \mathrm{P}$ & 0.45 \\
\hline PSOJ239.7124-07.4026 & 6.11 & $19.78 \pm 0.03$ & $2016-08-21$ & $12 \_1$ & $23.4 \mathrm{P}$ & 0.45 \\
\hline PSOJ242.4397-12.9816 & 5.83 & $19.76 \pm 0.04$ & 2014-02-04 & $1 \_1$ & $23.0 \mathrm{P}$ & 0.80 \\
\hline PSOJ245.0636-00.1978 & 5.68 & $21.15 \pm 0.07$ & 2015-05-19 & $1 \_1$ & $23.1 \mathrm{~S}$ & 0.26 \\
\hline PSOJ247.2970+24.1277 & 6.48 & $>22.77$ & 2017-05-01 & $12_{-}^{-} 1$ & $23.7 \mathrm{~S}$ & 0.14 \\
\hline PSOJ261.0364+19.0286 & 6.44 & $>22.92$ & $2017-04-22$ & $2 \_1$ & $23.8 \mathrm{P}$ & 0.12 \\
\hline PSOJ267.0021+22.7812 & 5.95 & $20.89 \pm 0.06$ & $2016-08-17$ & $1 \_1$ & $23.0 \mathrm{P}$ & 0.27 \\
\hline PSOJ308.0416-21.2339 & 6.24 & $21.12 \pm 0.08$ & 2014-09-24 & $1 \_12$ & $23.0 \mathrm{~S}$ & 0.14 \\
\hline PSOJ308.4829-27.6485 & 5.80 & $19.71 \pm 0.02$ & 2013-09-08 & $12 \_12$ & $23.0 \mathrm{P}$ & 0.13 \\
\hline
\end{tabular}


Table A.1. continued.

\begin{tabular}{|c|c|c|c|c|c|c|}
\hline Name & $\mathrm{z}$ & $\begin{array}{c}\mathrm{z}_{\text {cat }}^{\prime}(\mathrm{AB}) \\
(\mathrm{mag})\end{array}$ & Obs. date & OB & limiting $\mathrm{z}^{\prime}$ calib & $\begin{array}{c}\mathrm{A}_{\mathrm{V}} \\
(\mathrm{mag})\end{array}$ \\
\hline SDSSJ $2053+0047^{j}$ & 5.92 & $21.41 \pm 0.06$ & 2016-08-21 & $1 \_1$ & $23.3 \mathrm{~S}$ & 0.22 \\
\hline SDSSJ2054-0005 & 6.04 & $21.03 \pm 0.09$ & $2016-08-22$ & $1 \_1$ & $23.5 \mathrm{~S}$ & 0.26 \\
\hline CFHQSJ2100-1715 & 6.09 & $21.55 \pm 0.09$ & $2016-08-21$ & 1_1 & $23.6 \mathrm{~S}$ & 0.17 \\
\hline PSOJ319.6040-10.9326 & 5.90 & $19.94 \pm 0.02$ & $2013-09-10$ & $1 \_123$ & $23.4 \mathrm{P}$ & 0.13 \\
\hline SDSS211951.9-004020 ${ }^{c}$ & 5.87 & $21.68 \pm 0.10$ & 2017-09-21 & $\overline{1} \_1$ & $23.2 \mathrm{~S}$ & 0.12 \\
\hline PSOJ320.8703-24.3604 & 5.73 & $20.17 \pm 0.04$ & $2013-09-05$ & $1 \_123$ & $22.8 \mathrm{P}$ & 0.10 \\
\hline PSOJ323.1382+12.2986 ${ }^{d}$ & 6.59 & $21.56 \pm 0.10$ & 2017-04-27 & $\overline{1} \_1$ & $23.3 \mathrm{~S}$ & 0.28 \\
\hline SDSSJ $2147+0107^{j}$ & 5.81 & $21.61 \pm 0.08$ & $2016-08-22$ & $1 \_1$ & $23.3 \mathrm{~S}$ & 0.30 \\
\hline PSOJ328.7339-09.5076 & 5.92 & $20.82 \pm 0.06$ & $2016-08-22$ & $1 \_1$ & $23.2 \mathrm{P}$ & 0.12 \\
\hline IMSJ2204+0012 $2^{g}$ & 5.94 & $22.95 \pm 0.07$ & $2016-08-23$ & $1 \_1$ & $23.4 \mathrm{~S}$ & 0.13 \\
\hline HSCJ2216-0016 ${ }^{f}$ & 6.10 & $22.78 \pm 0.02$ & 2016-08-31 & $1 \_1$ & $23.7 \mathrm{~S}$ & 0.20 \\
\hline SDSSJ2220-0101 & 5.62 & $20.36 \pm 0.05$ & 2016-10-20 & $2 \_1$ & $23.4 \mathrm{~S}$ & 0.19 \\
\hline SDSSJ $2228+0110^{e}$ & 5.95 & $22.28 \pm 0.20$ & $2016-10-20$ & $2 \_1$ & $23.4 \mathrm{~S}$ & 0.18 \\
\hline CFHQSJ2229+1457 & 6.15 & $21.84 \pm 0.12$ & 2016-09-03 & 1_1 & $23.8 \mathrm{~S}$ & 0.15 \\
\hline PSOJ340.2041-18.6621 & 6.01 & $20.14 \pm 0.03$ & $2012-05-22$ & $1 \_123$ & $23.7 \mathrm{P}$ & 0.09 \\
\hline SDSSJ $2310+1855^{c}$ & 6.00 & $19.21 \pm 0.09$ & $2016-10-25$ & $\overline{1} \_1$ & $22.7 \mathrm{~S}$ & 0.46 \\
\hline CFHQSJ2329-0403 ${ }^{k}$ & 5.90 & $21.87 \pm 0.08$ & $2016-08-26$ & 1_1 & 23.0S & 0.12 \\
\hline PSOJ357.8289+06.4019 & 5.81 & $21.31 \pm 0.10$ & $2014-09-25$ & $1 \_1234$ & $23.2 \mathrm{~S}$ & 0.21 \\
\hline PSOJ359.1352-06.3831 & 6.15 & $19.97 \pm 0.03$ & $2014-09-25$ & $1 \_1$ & $22.6 \mathrm{~S}$ & 0.09 \\
\hline SDSSJ2356+0023 $3^{j}$ & 6.00 & $21.66 \pm 0.08$ & 2016-08-29 & $1 \_1$ & $23.2 \mathrm{~S}$ & 0.10 \\
\hline
\end{tabular}

Table A.2. Full GROND photometry of all 116 QSOs observed ${ }^{3}$.

\begin{tabular}{|c|c|c|c|c|c|c|c|}
\hline Name & $\begin{array}{c}g^{\prime} \\
(\mathrm{mag})\end{array}$ & $\begin{array}{c}r^{\prime} \\
(\mathrm{mag})\end{array}$ & $\begin{array}{c}i^{\prime} \\
\text { (mag) }\end{array}$ & $\begin{array}{c}z^{\prime} \\
\text { (mag) }\end{array}$ & $\begin{array}{c}J \\
\text { (mag) }\end{array}$ & $\begin{array}{c}H \\
\text { (mag) }\end{array}$ & $\begin{array}{c}K_{S} \\
(\mathrm{mag})\end{array}$ \\
\hline PSOJ000.3401+26.8358 & $>24.66$ & $22.92 \pm 0.15$ & $21.55 \pm 0.11$ & $19.43 \pm 0.04$ & $19.44 \pm 0.10$ & $19.10 \pm 0.13$ & $>19.19$ \\
\hline SDSSJ0005-0006 & $>24.95$ & $>24.55$ & $22.72 \pm 0.15$ & $20.54 \pm 0.04$ & $20.55 \pm 0.15$ & $20.35 \pm 0.19$ & $19.81 \pm 0.23$ \\
\hline PSOJ002.1073-06.4345 & $>23.62$ & $>23.62$ & $>22.70$ & $20.24 \pm 0.05$ & $19.82 \pm 0.11$ & $19.91 \pm 0.17$ & $>19.03$ \\
\hline PSOJ004.3936+17.0862 & $>24.62$ & $>24.09$ & $>22.59$ & $20.40 \pm 0.06$ & $20.26 \pm 0.20$ & $19.99 \pm 0.29$ & $>19.45$ \\
\hline PSOJ004.8140-24.2991 & $>23.36$ & $>22.85$ & $22.22 \pm 0.22$ & $19.55 \pm 0.04$ & $18.96 \pm 0.22$ & $19.01 \pm 0.13$ & $>17.87$ \\
\hline PSOJ007.0273+04.9571 & $>23.75$ & $>23.85$ & $22.64 \pm 0.20$ & $20.25 \pm 0.04$ & $19.91 \pm 0.13$ & $19.69 \pm 015$ & $>19.45$ \\
\hline CFHQSJ0033-0125 & $>24.45$ & $>23.95$ & $>23.33$ & $22.45 \pm 0.22$ & $21.10 \pm 0.24$ & $>20.90$ & $>19.05$ \\
\hline PSOJ009.3573-08.1190 & $>24.56$ & $22.94 \pm 0.26$ & $22.20 \pm 0.11$ & $20.09 \pm 0.04$ & $19.45 \pm 0.24$ & $>18.42$ & $>19.03$ \\
\hline PSOJ009.7355-10.4316 & $>24.42$ & & & $20.83 \pm 0.06$ & $19.56 \pm 0.10$ & $19.41 \pm 0.12$ & $19.43 \pm 0.20$ \\
\hline PSOJ011.3899+09.0325 & $>23.44$ & $>23.51$ & $>23.20$ & $>22.94$ & $>20.63$ & $>20.08$ & \\
\hline CFHQSJ0055+0146 & $>25.50$ & $>25.19$ & $>24.26$ & $22.41 \pm 0.09$ & $>21.96$ & $>21.44$ & $>20.04$ \\
\hline SDSSJ0100+2802 & $>24.80$ & $>24.53$ & $20.59 \pm 0.04$ & $18.27 \pm 0.03$ & $17.60 \pm 0.05$ & $17.49 \pm 0.05$ & $17.20 \pm 0.08$ \\
\hline CFHQSJ0102-0218 & $>22.83$ & $>23.34$ & $>22.98$ & $22.66 \pm 0.29$ & $>21.57$ & $>21.13$ & $>20.30$ \\
\hline PSOJ021.4213-25.8822 & $>23.91$ & $22.89 \pm 0.18$ & $22.36 \pm 0.19$ & $19.44 \pm 0.10$ & $19.19 \pm 0.07$ & $19.20 \pm 0.09$ & $19.20 \pm 0.17$ \\
\hline SDSSJ0129-0035 & $>25.13$ & $>24.80$ & $23.49 \pm 0.26$ & $22.24 \pm 0.14$ & $>21.42$ & $>20.54$ & $>19.70$ \\
\hline PSOJ023.0071-02.2675 & $>24.99$ & $>24.37$ & $23.39 \pm 0.28$ & $20.58 \pm 0.05$ & $19.85 \pm 0.09$ & $19.81 \pm 0.12$ & $19.28 \pm 0.30$ \\
\hline CFHQSJ0136+0226 & $>25.00$ & $>24.65$ & & $22.21 \pm 0.14$ & & & \\
\hline & & & & & & & \\
\hline ATLASJ025.6821-33.4627 & $>22.38$ & $>22.84$ & $21.32 \pm 0.25$ & $19.45 \pm 0.05$ & $18.86 \pm 0.06$ & $18.67 \pm 0.08$ & $18.57 \pm 0.21$ \\
\hline ULASJ0148+0600 & $>25.26$ & $>24.80$ & $22.51 \pm 0.09$ & $19.53 \pm 0.03$ & $19.19 \pm 0.06$ & $19.03 \pm 0.07$ & $18.78 \pm 0.14$ \\
\hline PSOJ029.5172-29.0886 & $>23.24$ & $>23.54$ & $21.94 \pm 0.09$ & $19.21 \pm 0.04$ & $19.12 \pm 0.07$ & $19.21 \pm 0.09$ & $18.42 \pm 0.16$ \\
\hline ATLASJ029.9915-36.565 & $>22.35$ & $>22.95$ & $21.71 \pm 0.20$ & $19.91 \pm 0.05$ & $>19.99$ & $19.43 \pm 0.13$ & $>19.99$ \\
\hline ULASJ0203+0012 & $>24.89$ & $>24.56$ & $>23.49$ & $20.57 \pm 0.05$ & $19.62 \pm 0.08$ & $19.22 \pm 0.08$ & $19.33 \pm 0.13$ \\
\hline CFHQSJ0210-0456 & $>25.09$ & $>24.75$ & $>23.82$ & $22.86 \pm 0.20$ & $>21.76$ & $>21.13$ & $>19.57$ \\
\hline CFHQSJ0221-0802 & $>22.72$ & $>23.34$ & $>22.93$ & $22.44 \pm 0.24$ & $>21.69$ & $>20.50$ & $>20.20$ \\
\hline PSOJ036.5078+03.0498 & $>24.68$ & $>24.34$ & $22.69 \pm 0.16$ & $20.54 \pm 0.05$ & $19.34 \pm 0.07$ & $19.61 \pm 0.10$ & $19.36 \pm 0.16$ \\
\hline CFHQSJ0227-0605 & $>25.45$ & & $>24.06$ & $22.74 \pm 0.17$ & $21.35 \pm 0.24$ & $>21.12$ & $>20.18$ \\
\hline PSOJ037.9706-28.8389 & $>25.13$ & $>24.77$ & $23.28 \pm 0.20$ & $20.65 \pm 0.04$ & $20.67 \pm 0.17$ & $>20.62$ & $>19.80$ \\
\hline
\end{tabular}

Notes. ${ }^{3}$ All magnitudes are in the AB system, and are corrected for the galactic extinction (see Table A.1). Upper limits are at the $3 \sigma$ confidence level. 
Table A.2. continued.

\begin{tabular}{|c|c|c|c|c|c|c|c|}
\hline Name & $\begin{array}{c}g^{\prime} \\
\text { (mag) }\end{array}$ & $\begin{array}{c}r^{\prime} \\
\text { (mag) }\end{array}$ & $\begin{array}{c}i^{\prime} \\
(\mathrm{mag}) \\
\end{array}$ & $\begin{array}{c}z^{\prime} \\
(\mathrm{mag})\end{array}$ & $\begin{array}{c}J \\
(\mathrm{mag})\end{array}$ & $\begin{array}{c}H \\
\text { (mag) }\end{array}$ & $\begin{array}{c}K_{s} \\
(\mathrm{mag})\end{array}$ \\
\hline SDSSJ0239-0045 & $>22.55$ & $>22.85$ & $>22.59$ & $>22.88$ & $>21.70$ & $>21.01$ & $>20.38$ \\
\hline PSOJ040.0159+17.5458 & $>24.94$ & $>24.24$ & $23.64 \pm 0.25$ & $20.87 \pm 0.04$ & $20.27 \pm 0.10$ & $20.28 \pm 0.14$ & $>19.91$ \\
\hline PSOJ042.6690-02.9174 & $>24.16$ & $>24.05$ & $23.28 \pm 0.28$ & $20.32 \pm 0.04$ & $20.37 \pm 0.12$ & $20.44 \pm 0.18$ & $>19.67$ \\
\hline PSOJ045.1840-22.5408 & $>25.29$ & $23.43 \pm 0.17$ & $22.40 \pm 0.14$ & $20.32 \pm 0.05$ & $19.68 \pm 0.10$ & $19.47 \pm 0.12$ & $19.23 \pm 0.21$ \\
\hline SDSSJ0303-0019 & $>25.36$ & $>25.29$ & $>23.98$ & $21.17 \pm 0.05$ & $20.92 \pm 0.15$ & $>20.92$ & $>19.98$ \\
\hline VIKJ0305-3150 & $>24.99$ & $>21.66$ & $>23.99$ & $21.96 \pm 0.18$ & $21.32 \pm 0.28$ & $>20.82$ & $>18.50$ \\
\hline CFHQSJ0316-1340 & $>25.43$ & $>25.21$ & $23.98 \pm 0.27$ & $21.43 \pm 0.05$ & $20.79 \pm 0.22$ & $20.65 \pm 0.26$ & $>20.39$ \\
\hline PSOJ049.2934-26.5543 & $>24.85$ & $>24.47$ & $>23.80$ & $20.72 \pm 0.05$ & $21.39 \pm 0.27$ & $>21.26$ & $>18.84$ \\
\hline VIKJ0328-3253 & $>23.39$ & $>23.57$ & $22.77 \pm 0.23$ & $20.17 \pm 0.04$ & $20.14 \pm 0.10$ & $19.98 \pm 0.14$ & $>19.49$ \\
\hline PSOJ053.9605-15.7956 & $>23.52$ & $>23.57$ & $>22.96$ & $20.84 \pm 0.05$ & $20.77 \pm 0.18$ & $20.69 \pm 0.28$ & $19.74 \pm 0.28$ \\
\hline PSOJ055.4244-00.8035 & $>23.41$ & $>23.59$ & $22.00 \pm 0.19$ & $20.51 \pm 0.07$ & $20.12 \pm 0.23$ & $20.04 \pm 0.26$ & $>19.28$ \\
\hline PSOJ056.7168-16.4769 & $>22.25$ & $>22.63$ & $>22.45$ & $19.92 \pm 0.04$ & $19.55 \pm 0.10$ & $20.07 \pm 0.28$ & $19.40 \pm 0.28$ \\
\hline SDSSJ0353+0104 & $>23.83$ & $>24.82$ & $>23.80$ & $20.86 \pm 0.06$ & $19.76 \pm 0.11$ & $20.06 \pm 0.17$ & $19.11 \pm 0.21$ \\
\hline PSOJ060.5529+24.8567 & $>24.53$ & $>24.30$ & $>23.38$ & $19.94 \pm 0.04$ & $19.64 \pm 0.12$ & $19.55 \pm 0.17$ & $19.41 \pm 0.26$ \\
\hline PSOJ065.4085-26.9543 & $>23.74$ & $>23.91$ & $22.43 \pm 0.24$ & $20.15 \pm 0.05$ & $19.04 \pm 0.10$ & $19.10 \pm 0.10$ & $>18.94$ \\
\hline PSOJ065.5041-19.4579 & $>24.00$ & $>23.83$ & $>22.85$ & $19.80 \pm 0.04$ & $20.03 \pm 0.24$ & $19.08 \pm 0.12$ & $19.16 \pm 0.23$ \\
\hline PSOJ071.0322-04.5591 & $>25.19$ & $>24.54$ & $22.88 \pm 0.13$ & $20.08 \pm 0.03$ & $20.26 \pm 0.10$ & $20.22 \pm 0.14$ & $19.85 \pm 0.19$ \\
\hline PSOJ071.4507-02.3332 & $>24.95$ & $22.27 \pm 0.05$ & $21.47 \pm 0.05$ & $19.10 \pm 0.03$ & $18.97 \pm 0.06$ & $18.95 \pm 0.06$ & $19.11 \pm 0.16$ \\
\hline DESJ0454-4448 & $>23.73$ & $>24.18$ & $22.77 \pm 0.15$ & $20.54 \pm 0.04$ & $20.32 \pm 0.12$ & $19.95 \pm 0.15$ & $19.64 \pm 0.30$ \\
\hline PSOJ075.9356-07.5061 & $>24.94$ & $>24.66$ & $23.32 \pm 0.26$ & $20.50 \pm 0.04$ & $20.34 \pm 0.15$ & $20.37 \pm 0.21$ & $>19.31$ \\
\hline PSOJ089.9394-15.5833 & $>24.87$ & $>24.64$ & $22.81 \pm 0.22$ & $19.45 \pm 0.03$ & $18.84 \pm 0.06$ & $18.51 \pm 0.06$ & $18.28 \pm 0.09$ \\
\hline PSOJ108.4429+08.9257 & $>24.40$ & $>24.04$ & $22.36 \pm 0.19$ & $19.21 \pm 0.03$ & $19.15 \pm 0.08$ & $18.94 \pm 0.09$ & $18.89 \pm 0.14$ \\
\hline SDSSJ0818+1722 & $>24.23$ & $>24.12$ & $21.71 \pm 0.09$ & $19.54 \pm 0.04$ & $19.04 \pm 0.09$ & $18.59 \pm 0.09$ & $>17.46$ \\
\hline ULASJ0828+2633 & $>22.64$ & $>23.11$ & $>22.58$ & $20.58 \pm 0.08$ & $20.07 \pm 0.17$ & $20.10 \pm 0.25$ & $>18.57$ \\
\hline PSOJ127.2817+03.0657 & $>24.09$ & $>23.75$ & $>23.29$ & $20.69 \pm 0.06$ & $20.73 \pm 0.23$ & $20.13 \pm 0.22$ & $19.76 \pm 0.24$ \\
\hline SDSSJ0836+0054 & $>24.64$ & $22.20 \pm 0.05$ & $21.18 \pm 0.05$ & $18.75 \pm 0.03$ & $18.51 \pm 0.06$ & $18.17 \pm 0.06$ & $17.99 \pm 0.09$ \\
\hline VIKJ0839+0015 & $>24.49$ & $>24.34$ & $>23.56$ & $21.51 \pm 0.08$ & $20.61 \pm 0.29$ & $>20.68$ & $>18.15$ \\
\hline SDSSJ0842+1218 & $>22.39$ & $>22.55$ & $>21.71$ & $19.79 \pm 0.07$ & $19.48 \pm 0.11$ & $19.35 \pm 0.13$ & $18.94 \pm 0.29$ \\
\hline HSCJ0859+0022 & $>24.60$ & $>24.52$ & $>23.70$ & $23.06 \pm 0.27$ & $>20.11$ & $>19.81$ & $>17.41$ \\
\hline PSOJ135.3860+16.2518 & $>24.20$ & $>23.91$ & $22.79 \pm 0.27$ & $20.73 \pm 0.07$ & $20.51 \pm 0.18$ & $>20.49$ & $>19.72$ \\
\hline PSOJ135.8704-13.8336 & $>24.86$ & $>24.36$ & $23.20 \pm 0.23$ & $20.25 \pm 0.04$ & $20.88 \pm 0.22$ & $20.42 \pm 0.25$ & $>19.93$ \\
\hline PSOJ157.9070-02.6599 & $>24.23$ & $22.28 \pm 0.09$ & $22.53 \pm 0.15$ & $20.16 \pm 0.04$ & $20.03 \pm 0.12$ & $20.38 \pm 0.22$ & $>19.23$ \\
\hline PSOJ159.2257-02.5438 & $>24.85$ & $>24.63$ & $>23.93$ & $20.41 \pm 0.05$ & $19.74 \pm 0.12$ & $19.81 \pm 0.18$ & $>18.65$ \\
\hline SDSSJ1044-0125 & $>24.74$ & $22.80 \pm 0.08$ & $21.82 \pm 0.07$ & $19.19 \pm 0.03$ & $18.94 \pm 0.07$ & $18.88 \pm 0.08$ & $>18.58$ \\
\hline CFHQSJ1059-0906 & $>25.25$ & $>24.81$ & $>23.57$ & $20.74 \pm 0.04$ & $20.34 \pm 0.15$ & $20.35 \pm 0.20$ & $>18.36$ \\
\hline PSOJ167.6415-13.4960 & $>24.54$ & $>24.44$ & $>23.66$ & $22.12 \pm 0.13$ & $20.70 \pm 0.21$ & $20.28 \pm 0.23$ & $>19.46$ \\
\hline PSOJ174.7920-12.2845 & $>24.87$ & $23.88 \pm 0.16$ & $22.66 \pm 0.14$ & $20.25 \pm 0.04$ & $20.37 \pm 0.19$ & $>20.25$ & $>19.99$ \\
\hline PSOJ175.4091-20.2654 & $>25.02$ & $23.95 \pm 0.21$ & $22.86 \pm 0.14$ & $20.29 \pm 0.04$ & $19.80 \pm 0.10$ & $19.59 \pm 0.10$ & $>18.68$ \\
\hline VIKJ1152+0055 & $>24.90$ & $>24.72$ & $>23.90$ & $22.30 \pm 0.15$ & $21.18 \pm 0.28$ & $>21.16$ & $>20.21$ \\
\hline PSOJ183.2991-12.7676 & $>24.77$ & $23.49 \pm 0.13$ & $21.79 \pm 0.07$ & $18.81 \pm 0.04$ & $19.02 \pm 0.06$ & $18.80 \pm 0.07$ & $18.99 \pm 0.22$ \\
\hline PSOJ184.3389+01.5284 & $>24.35$ & $>24.40$ & $>23.61$ & $21.29 \pm 0.07$ & $>20.98$ & $>20.62$ & $>19.36$ \\
\hline PSOJ187.1047-02.5609 & $>24.32$ & $>23.94$ & $>23.18$ & $21.02 \pm 0.07$ & $20.70 \pm 0.18$ & $20.41 \pm 0.21$ & $>20.06$ \\
\hline PSOJ187.3050+04.3243 & $>24.82$ & $>24.55$ & $>23.79$ & $21.16 \pm 0.06$ & $20.94 \pm 0.29$ & $>20.68$ & $>19.64$ \\
\hline SDSSJ1306+0356 & $>24.84$ & $>24.48$ & $23.04 \pm 0.21$ & $19.71 \pm 0.04$ & $>19.02$ & $>19.15$ & $>17.61$ \\
\hline ULASJ1319+0950 & $>25.31$ & $>24.50$ & $22.74 \pm 0.12$ & $20.16 \pm 0.04$ & $19.60 \pm 0.08$ & $19.53 \pm 0.11$ & $>19.50$ \\
\hline PSOJ209.2058-26.7083 & $>24.39$ & $>23.57$ & $21.98 \pm 0.10$ & $19.41 \pm 0.03$ & $19.20 \pm 0.08$ & $19.20 \pm 0.10$ & $19.32 \pm 0.22$ \\
\hline PSOJ210.8297+09.0474 & $>24.26$ & $>23.60$ & $>22.74$ & $20.39 \pm 0.07$ & $>20.17$ & $>20.02$ & $>19.99$ \\
\hline PSOJ210.8722-12.0094 & $>25.01$ & $>24.64$ & $>23.30$ & $21.04 \pm 0.06$ & $20.96 \pm 0.23$ & $>20.27$ & $>19.78$ \\
\hline PSOJ212.2974-15.9865 & $>24.61$ & $>24.24$ & $>23.74$ & $21.20 \pm 0.07$ & $20.53 \pm 0.16$ & $20.34 \pm 0.19$ & $>20.03$ \\
\hline SDSSJ1411+1217 & $>24.07$ & $>23.49$ & $>22.82$ & $19.68 \pm 0.05$ & $20.09 \pm 0.20$ & $19.48 \pm 0.23$ & $>19.25$ \\
\hline PSOJ213.3629-22.5617 & $>24.41$ & $>24.17$ & $>23.14$ & $19.73 \pm 0.04$ & $19.94 \pm 0.12$ & $20.04 \pm 0.21$ & $>18.99$ \\
\hline PSOJ213.7329-13.4803 & $>24.39$ & $>23.97$ & $22.94 \pm 0.29$ & $20.95 \pm 0.07$ & $20.70 \pm 0.26$ & $20.20 \pm 0.28$ & $>19.37$ \\
\hline PSOJ215.1514-16.0417 & $>24.50$ & $22.85 \pm 0.12$ & $22.26 \pm 0.13$ & $19.07 \pm 0.03$ & $18.79 \pm 0.06$ & $18.61 \pm 0.07$ & $18.59 \pm 0.26$ \\
\hline PSOJ217.0891-16.0453 & $>24.34$ & $>24.16$ & $22.46 \pm 0.21$ & $20.29 \pm 0.05$ & $19.59 \pm 0.10$ & $19.97 \pm 0.19$ & $>19.43$ \\
\hline PSOJ217.9185-07.4120 & $>24.90$ & $>24.49$ & $23.41 \pm 0.24$ & $20.74 \pm 0.04$ & $19.79 \pm 0.08$ & $19.73 \pm 0.11$ & $19.77 \pm 0.21$ \\
\hline CFHQSJ1509-1749 & $>23.17$ & $>23.73$ & $>23.02$ & $20.05 \pm 0.04$ & $19.71 \pm 0.09$ & $19.66 \pm 0.16$ & $19.69 \pm 0.27$ \\
\hline
\end{tabular}


Table A.2. continued.

\begin{tabular}{|c|c|c|c|c|c|c|c|}
\hline Name & $\begin{array}{c}g^{\prime} \\
(\mathrm{mag})\end{array}$ & $\begin{array}{c}r^{\prime} \\
\text { (mag) }\end{array}$ & $\begin{array}{c}i^{\prime} \\
(\mathrm{mag})\end{array}$ & $\begin{array}{c}z^{\prime} \\
\text { (mag) }\end{array}$ & $\begin{array}{c}J \\
\text { (mag) }\end{array}$ & $\begin{array}{c}H \\
(\mathrm{mag})\end{array}$ & $\begin{array}{c}K_{s} \\
(\mathrm{mag})\end{array}$ \\
\hline PSOJ228.6871+21.2388 & $>25.34$ & $>24.92$ & $>23.65$ & $20.64 \pm 0.04$ & $20.51 \pm 0.17$ & $20.19 \pm 0.21$ & $>19.16$ \\
\hline PSOJ235.9450+17.0079 & $>24.77$ & $23.52 \pm 0.14$ & $22.64 \pm 0.16$ & $20.35 \pm 0.04$ & $20.21 \pm 0.12$ & $19.92 \pm 0.18$ & $>19.47$ \\
\hline PSOJ236.2912+16.6088 & $>24.91$ & $>23.96$ & $>23.50$ & $21.04 \pm 0.08$ & $>20.70$ & $>20.09$ & $>20.15$ \\
\hline PSOJ238.8510-06.8976 & $>24.13$ & $>23.92$ & $>22.91$ & $20.32 \pm 0.06$ & $20.11 \pm 0.18$ & $20.16 \pm 0.25$ & $>19.94$ \\
\hline PSOJ239.7124-07.4026 & $>24.95$ & $>23.50$ & $22.33 \pm 0.15$ & $19.69 \pm 0.03$ & $19.27 \pm 0.09$ & $19.22 \pm 0.11$ & $18.92 \pm 0.18$ \\
\hline PSOJ242.4397-12.9816 & $>23.91$ & $>23.15$ & $>22.42$ & $19.72 \pm 0.04$ & $19.42 \pm 0.11$ & $19.49 \pm 0.16$ & $19.21 \pm 0.28$ \\
\hline PSOJ245.0636-00.1978 & $>24.34$ & $>24.10$ & $>23.38$ & $21.57 \pm 0.11$ & $20.83 \pm 0.27$ & $>20.57$ & $>19.77$ \\
\hline PSOJ247.2970+24.1277 & $>25.59$ & $>25.26$ & $>24.06$ & $21.07 \pm 0.05$ & $20.02 \pm 0.10$ & $19.86 \pm 0.15$ & $>19.45$ \\
\hline PSOJ261.0364+19.0286 & $>25.02$ & $>24.69$ & $23.96 \pm 0.26$ & $22.12 \pm 0.09$ & $20.72 \pm 0.17$ & $20.39 \pm 0.17$ & $>19.94$ \\
\hline PSOJ267.0021+22.7812 & $>22.73$ & $>23.28$ & $>22.97$ & $21.76 \pm 0.19$ & $>20.75$ & $>20.36$ & $>19.91$ \\
\hline PSOJ308.0416-21.2339 & $>24.66$ & $>24.35$ & $>23.28$ & $20.47 \pm 0.05$ & $20.03 \pm 0.11$ & $19.63 \pm 0.12$ & $19.47 \pm 0.17$ \\
\hline PSOJ308.4829-27.6485 & $>23.95$ & $23.56 \pm 0.28$ & $22.22 \pm 0.13$ & $19.66 \pm 0.04$ & $19.46 \pm 0.09$ & $19.39 \pm 0.12$ & $18.87 \pm 0.19$ \\
\hline SDSSJ2053+0047 & $>23.50$ & $>23.71$ & $>23.31$ & $21.21 \pm 0.07$ & $>20.93$ & $20.73 \pm 0.25$ & $>19.92$ \\
\hline SDSSJ2054-0005 & $>23.67$ & $>24.02$ & $>23.01$ & $20.67 \pm 0.04$ & $20.33 \pm 0.11$ & $20.04 \pm 0.15$ & $19.31 \pm 0.22$ \\
\hline CFHQSJ2100-1715 & $>23.99$ & $>24.16$ & $>23.73$ & $21.45 \pm 0.06$ & $20.45 \pm 0.12$ & $19.91 \pm 0.12$ & $19.84 \pm 0.23$ \\
\hline PSOJ319.6040-10.9326 & $>24.30$ & $>24.43$ & $22.41 \pm 0.12$ & $19.99 \pm 0.03$ & $19.96 \pm 0.09$ & $19.72 \pm 0.11$ & $19.47 \pm 0.30$ \\
\hline SDSS211951.9-004020 & $>24.75$ & $>24.66$ & $>23.45$ & $22.30 \pm 0.17$ & $>20.65$ & $>20.08$ & $>18.72$ \\
\hline PSOJ320.8703-24.3604 & $>23.89$ & $>24.05$ & $22.84 \pm 0.27$ & $20.27 \pm 0.05$ & $20.19 \pm 0.12$ & $19.42 \pm 0.10$ & $>19.64$ \\
\hline PSOJ323.1382+12.2986 & $>23.79$ & $>24.58$ & $23.40 \pm 0.26$ & $20.29 \pm 0.04$ & $19.32 \pm 0.07$ & $19.20 \pm 0.10$ & $19.07 \pm 0.22$ \\
\hline SDSSJ $2147+0107$ & $>23.62$ & $>24.05$ & $>23.45$ & $21.71 \pm 0.10$ & $>21.27$ & $>20.80$ & $>19.69$ \\
\hline PSOJ328.7339-09.5076 & $>23.70$ & $>24.03$ & $>23.14$ & $20.58 \pm 0.05$ & $20.13 \pm 0.12$ & $20.00 \pm 0.15$ & $>19.47$ \\
\hline IMSJ2204+0012 & $>23.58$ & $>24.02$ & $>23.16$ & $22.96 \pm 0.23$ & $>21.34$ & $>20.77$ & $>19.69$ \\
\hline HSCJ2216-0016 & $>25.22$ & $>25.18$ & $>24.25$ & $22.91 \pm 0.19$ & $>21.87$ & $>21.25$ & $>19.98$ \\
\hline SDSSJ2220-0101 & $>24.83$ & $>23.73$ & $22.18 \pm 0.10$ & $20.82 \pm 0.05$ & $20.34 \pm 0.14$ & $20.07 \pm 0.16$ & $>20.28$ \\
\hline SDSSJ2228+0110 & $>25.73$ & $>24.51$ & $>23.64$ & $22.18 \pm 0.14$ & $21.28 \pm 0.28$ & $>20.71$ & $>18.92$ \\
\hline CFHQSJ2229+1457 & $>25.19$ & $>24.96$ & $>23.36$ & $21.87 \pm 0.08$ & $>21.24$ & $>21.04$ & $>20.60$ \\
\hline PSOJ340.2041-18.6621 & $>25.25$ & $>25.00$ & $23.56 \pm 0.22$ & $20.17 \pm 0.03$ & $20.05 \pm 0.09$ & $19.91 \pm 0.15$ & $19.60 \pm 0.24$ \\
\hline SDSSJ2310+1855 & $>24.71$ & $>24.25$ & $21.76 \pm 0.13$ & $19.24 \pm 0.04$ & $18.63 \pm 0.06$ & $18.73 \pm 0.10$ & $>19.99$ \\
\hline CFHQSJ2329-0403 & $>24.00$ & $>24.30$ & $>23.36$ & $21.90 \pm 0.17$ & $>20.21$ & $>19.59$ & $>17.72$ \\
\hline PSOJ357.8289+06.4019 & $>24.57$ & $>23.78$ & $>23.66$ & $21.43 \pm 0.09$ & $>21.12$ & $>20.85$ & $>20.02$ \\
\hline PSOJ359.1352-06.3831 & $>23.87$ & $>23.57$ & $>22.36$ & $19.90 \pm 0.05$ & $19.89 \pm 0.15$ & $19.76 \pm 0.20$ & $18.91 \pm 0.21$ \\
\hline SDSSJ $2356+0023$ & $>24.54$ & $>24.23$ & $>23.46$ & $>23.10$ & $>20.66$ & $>20.31$ & $>19.54$ \\
\hline
\end{tabular}

Table A.3. Log of spectroscopic observations of QSO pair candidates in 2019. All are 900 sec exposures with grating R150 and a 0"'9 slit, except for the FIRE spectrum ( $1^{\prime \prime}$ slit).

\begin{tabular}{lcl}
\hline \hline QSO candidate & $\begin{array}{c}\text { Date / Time } \\
\text { (MM-DD HH:MM) }\end{array}$ & $\begin{array}{l}\text { Instrument/ } \\
\text { Telescope }\end{array}$ \\
\hline IMSJ2204+0012 & 06-26 08:01 & FIRE/Baade \\
SDSSJ2054-0005_4 & $09-03$ 01:34 & GMOS-S \\
SDSSJ2054-0005_3 & $09-0302: 04$ & GMOS-S \\
PSOJ261.0364+19.0286 & $09-0308: 59$ & GMOS-N \\
PSOJ267.0021+22.7812 & $09-0309: 30$ & GMOS-N \\
CFHQSJ1509-1749 & $09-0400: 03$ & GMOS-S \\
PSOJ011.3899+09.0325 & $09-0614: 22$ & GMOS-N \\
ULASJ0148+0600 & $09-0614: 52$ & GMOS-N \\
PSOJ009.7355-10.4316 & $09-1905: 34$ & GMOS-S \\
PSOJ045.1840-22.5408 & $09-2204: 38$ & GMOS-S \\
PSOJ071.0322-04.5591 & $10-2905: 29$ & GMOS-S \\
\hline
\end{tabular}


Table A.4. Pair candidates with existing ALLWISE channel W1/W2 photometry.

\begin{tabular}{|c|c|c|c|c|c|c|c|c|c|c|c|}
\hline Prime QSO & RA/Decl. (2000.0) & $g^{\prime}$ & $r^{\prime}$ & $i^{\prime}$ & $z^{\prime}$ & $J$ & $H$ & $K$ & W1 & W2 & ID \\
\hline CFHQSJ0227-0605 & $36.9473-06.0495$ & $>25.56$ & $24.26 \pm 0.17$ & $21.64 \pm 0.05$ & $20.11 \pm 0.04$ & $18.91 \pm 0.06$ & $18.65 \pm 0.06$ & $18.84 \pm 0.13$ & $19.67 \pm 0.10$ & $20.38 \pm 0.33$ & L0 \\
\hline HSCJ0859+0022 & $134.7528+00.3927$ & $>24.90$ & $23.30 \pm 0.10$ & $20.88 \pm 0.04$ & $19.29 \pm 0.03$ & $18.05 \pm 0.06$ & $17.82 \pm 0.06$ & $>17.72$ & $18.67 \pm 0.05$ & $19.26 \pm 0.16$ & L0 \\
\hline PSOJ037.9706-28.8389 & $38.0071-28.8519$ & $>25.02$ & $>24.59$ & $23.27 \pm 0.21$ & $21.75 \pm 0.08$ & $20.27 \pm 0.12$ & $19.99 \pm 0.14$ & $19.70 \pm 0.23$ & $20.29 \pm 0.15$ & - & M9 \\
\hline PSOJ055.4244-00.8035 & $55.4110-00.8079$ & $>23.72$ & $>23.52$ & $>23.25$ & $21.71 \pm 0.19$ & $20.08 \pm 0.24$ & $>20.26$ & $>19.21$ & $20.04 \pm 0.15$ & $19.95 \pm 0.30$ & QSO \\
\hline PSOJ075.9356-07.5061 & $75.9660-07.4760$ & $>25.59$ & $>25.11$ & $22.03 \pm 0.07$ & $20.43 \pm 0.04$ & $19.27 \pm 0.07$ & $18.95 \pm 0.07$ & $18.97 \pm 0.28$ & $19.64 \pm 0.11$ & $>19.93$ & L3 \\
\hline PSOJ135.8704-13.8336 & $135.8657-13.8220$ & $>25.08$ & $23.44 \pm 0.11$ & $21.13 \pm 0.04$ & $19.43 \pm 0.03$ & $17.91 \pm 0.05$ & $17.62 \pm 0.05$ & $17.86 \pm 0.08$ & $18.29 \pm 0.04$ & $18.78 \pm 0.10$ & L3 \\
\hline PSOJ340.2041-18.6621 & $340.2045-18.6680$ & $>25.36$ & $>24.87$ & $22.09 \pm 0.06$ & $20.53 \pm 0.04$ & $19.08 \pm 0.06$ & $18.81 \pm 0.07$ & $19.14 \pm 0.15$ & $19.65 \pm 0.12$ & $19.77 \pm 0.26$ & L1 \\
\hline SDSSJ0005-0006 & $1.4755-00.0968$ & $>25.06$ & $23.76 \pm 0.18$ & $21.40 \pm 0.05$ & $19.67 \pm 0.03$ & $18.14 \pm 0.05$ & $17.94 \pm 0.06$ & $17.88 \pm 0.08$ & $18.67 \pm 0.06$ & $18.79 \pm 0.11$ & M9 \\
\hline ULASJ0148+0600 & $27.1558+06.0265$ & $>25.46$ & $>24.89$ & $>23.92$ & $22.41 \pm 0.13$ & $21.06 \pm 0.18$ & $20.93 \pm 0.27$ & $>19.28$ & $19.90 \pm 0.14$ & $>19.89$ & $\mathrm{QSO} / \mathrm{Gal}$ \\
\hline
\end{tabular}

Notes. Magnitudes are not corrected for foreground $A_{v}$, all in the AB system. The ID column gives the best-fitting Le PHARE spectral template. The object marked in boldface had a QSO template as best-fit Le PHARE output, which is inconsistent with our GMOS spectrum; we therefore provide the next best-fit template, a low-redshift galaxy, as potential identification.

Table A.5. Pair candidates without ALLWISE photometry.

\begin{tabular}{|c|c|c|c|c|c|c|c|c|c|}
\hline Prime QSO & RA/Decl. (2000.0) & $g^{\prime}$ & $r^{\prime}$ & $i^{\prime}$ & $z^{\prime}$ & $J$ & $H$ & $K$ & ID \\
\hline CFHQSJ1509-1749 & $227.4413-17.8303$ & $>23.47$ & $>23.94$ & $>23.32$ & $22.31 \pm 0.16$ & $21.28 \pm 0.27$ & $>21.01$ & $>20.32$ & QSO/M8 \\
\hline DESJ0454-4448 & $73.5198-44.8196$ & $>23.65$ & $>24.21$ & $23.49 \pm 0.26$ & $21.60 \pm 0.08$ & $20.05 \pm 0.10$ & $20.41 \pm 0.20$ & $19.70 \pm 0.29$ & L1 \\
\hline IMSJ2204+0012 & $331.0878+01.2344$ & $>23.79$ & $>24.20$ & $>23.66$ & $22.09 \pm 0.11$ & $20.95 \pm 0.21$ & $20.37 \pm 0.21$ & $>19.71$ & QSO/M8 \\
\hline PSOJ009.7355-10.4316 & $9.7185-10.4161$ & $>24.52$ & $>24.36$ & $>23.17$ & $21.79 \pm 0.12$ & $20.85 \pm 0.28$ & $20.54 \pm 0.30$ & $>19.99$ & QSO/M9 \\
\hline PSOJ011.3899+09.0325 & $11.3462+09.0813$ & $>23.84$ & $>23.84$ & $>23.51$ & $21.54 \pm 0.09$ & $>21.67$ & $20.96 \pm 0.33$ & $>20.13$ & QSO/M6 \\
\hline PSOJ036.5078+03.0498 & $36.5245+03.0958$ & $>24.79$ & $>24.69$ & $23.30 \pm 0.28$ & $21.60 \pm 0.09$ & $20.06 \pm 0.10$ & $20.02 \pm 0.14$ & $>20.08$ & * \\
\hline PSOJ045.1840-22.5408 & $45.1936-22.5280$ & $>25.37$ & $>24.59$ & $>23.60$ & $21.97 \pm 0.12$ & $20.66 \pm 0.19$ & $20.16 \pm 0.20$ & $>19.33$ & QSO/M9 \\
\hline PSOJ071.0322-04.5591 & $71.0745-04.5348$ & $>24.87$ & $>24.38$ & $>24.08$ & $22.29 \pm 0.13$ & $21.22 \pm 0.20$ & $>20.94$ & $19.74 \pm 0.18$ & $*$ \\
\hline PSOJ071.4507-02.3332 & $71.4815-02.3637$ & $>25.08$ & $>24.75$ & $23.25 \pm 0.18$ & $21.65 \pm 0.07$ & $20.45 \pm 0.11$ & $20.52 \pm 0.18$ & $>20.11$ & $*$ \\
\hline PSOJ261.0364+19.0286 & $261.0269+19.0228$ & $>25.23$ & $>24.85$ & $23.90 \pm 0.23$ & $22.23 \pm 0.09$ & $>21.48$ & $>20.84$ & $>19.89$ & QSO/? \\
\hline PSOJ267.0021+22.7812 & $266.9979+22.7922$ & $>23.07$ & $>23.51$ & $>23.14$ & $21.35 \pm 0.09$ & $20.85 \pm 0.23$ & $20.28 \pm 0.25$ & $>19.86$ & QSO/T6 \\
\hline PSOJ328.7339-09.5076 & $328.7633-09.4779$ & $>23.80$ & $>24.10$ & $23.25 \pm 0.32$ & $21.63 \pm 0.09$ & $20.44 \pm 0.14$ & $20.03 \pm 0.13$ & $>19.38$ & $*$ \\
\hline SDSSJ0303-0019 & $45.8859-00.3281$ & $>25.72$ & $>25.16$ & $>24.49$ & $22.89 \pm 0.14$ & $>22.02$ & $>21.53$ & $>20.06$ & $\mathrm{~T} 8$ \\
\hline SDSSJ2053+0047_1 & $313.3294+00.7677$ & $>23.53$ & $>23.91$ & $>23.41$ & $22.20 \pm 0.14$ & $21.01 \pm 0.25$ & $21.04 \pm 0.33$ & $>19.99$ & M9 \\
\hline SDSSJ2053+0047_3 & $313.3766+00.7606$ & $>23.78$ & $>23.70$ & $>23.49$ & $22.06 \pm 0.15$ & $21.34 \pm 0.30$ & $>20.91$ & $>20.34$ & M5 \\
\hline SDSSJ2054-0005_3 & $313.5334-00.0914$ & $>24.00$ & $>24.25$ & $>23.71$ & $21.81 \pm 0.10$ & $20.82 \pm 0.18$ & $20.44 \pm 0.25$ & $>19.73$ & $*$ \\
\hline SDSSJ2054-0005_4 & $313.5450-00.0525$ & $>24.00$ & $>24.25$ & $>23.71$ & $22.16 \pm 0.12$ & $20.98 \pm 0.20$ & $20.79 \pm 0.28$ & $>19.98$ & $*$ \\
\hline
\end{tabular}

Notes. Magnitudes are not corrected for foreground $A_{v}$, all in the AB system. The ID column gives the best-fitting Le PHARE spectral template. The objects marked in boldface are those where the Le PHARE fit preferred a QSO high-z template, and we obtained optical spectroscopy. Except for PSOJ071.0322-04.5591, our spectra argue against a QSO nature, and we therefore provide the next best-fitting templates, mostly dwarf stars (see text). For objects with an asterisk (*) in the last column, see Table A.6.

Table A.6. Pair candidates from Table A.5 with unWISE or CatWISE photometry (AB system).

\begin{tabular}{rccccll}
\hline \hline Prime QSO & \multicolumn{2}{c}{ unWISE } & \multicolumn{2}{c}{ CatWISE } & ID1 & \multirow{2}{*}{ ID2 } \\
& $\mathrm{W} 1$ & $\mathrm{~W} 2$ & $\mathrm{~W} 1$ & $\mathrm{~W} 2$ & & \\
\hline PSOJ036.5078+03.0498 & - & - & $20.96 \pm 0.16$ & $21.03 \pm 0.32$ & L2 & L0 \\
PSOJ071.0322-04.5591 & $19.47 \pm 0.04$ & $19.57 \pm 0.09$ & $19.39 \pm 0.04$ & $19.63 \pm 0.09$ & QSO & low-z Gal \\
PSOJ071.4507-02.3332 & $21.09 \pm 0.04$ & - & $21.37 \pm 0.21$ & $21.45 \pm 0.45$ & M9 & M9 \\
PSOJ328.7339-09.5076 & $19.06 \pm 0.05$ & $19.26 \pm 0.10$ & $19.13 \pm 0.04$ & $19.31 \pm 0.07$ & M8 & low-z Gal \\
SDSSJ2054-0005_3 & $19.73 \pm 0.08$ & - & $19.80 \pm 0.06$ & $20.46 \pm 0.17$ & QSO & $?$ \\
SDSSJ2054-0005_4 & - & - & $21.06 \pm 0.18$ & $21.15 \pm N U L L$ & QSO & M8 \\
\hline
\end{tabular}

Notes. The ID columns give the best-fitting Le PHARE spectral template without (ID1) and with un/Cal-WISE photometry (ID2). 